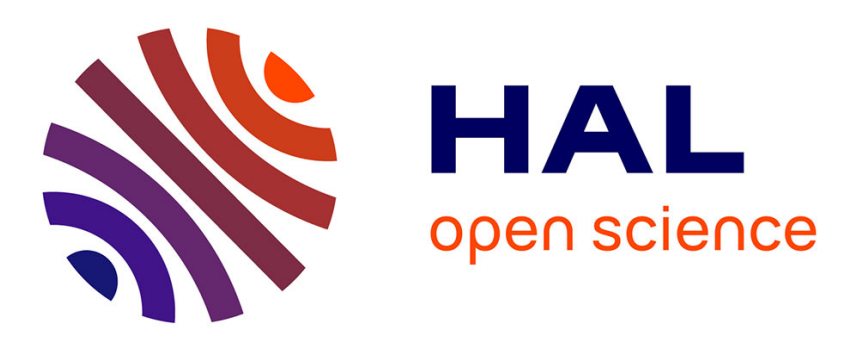

\title{
A low cost semi-implicit low-Mach relaxation scheme for the full Euler equations
}

\author{
François Bouchut, Emmanuel Franck, Laurent Navoret
}

\section{To cite this version:}

François Bouchut, Emmanuel Franck, Laurent Navoret. A low cost semi-implicit low-Mach relaxation scheme for the full Euler equations. Journal of Scientific Computing, 2020, 83 (1), pp.24. 10.1007/s10915-020-01206-z . hal-02420859v3

\section{HAL Id: hal-02420859 \\ https://hal.science/hal-02420859v3}

Submitted on 23 Mar 2020

HAL is a multi-disciplinary open access archive for the deposit and dissemination of scientific research documents, whether they are published or not. The documents may come from teaching and research institutions in France or abroad, or from public or private research centers.
L'archive ouverte pluridisciplinaire HAL, est destinée au dépôt et à la diffusion de documents scientifiques de niveau recherche, publiés ou non, émanant des établissements d'enseignement et de recherche français ou étrangers, des laboratoires publics ou privés. 


\title{
A low cost semi-implicit low-Mach relaxation scheme for the full Euler equations
}

\author{
François Bouchut ${ }^{1}$, Emmanuel Franck ${ }^{2,3}$, Laurent Navoret ${ }^{3}$
}

March 23, 2020

${ }^{1}$ Laboratoire d'Analyse et de Mathématiques Appliquées (UMR 8050), CNRS, Univ. Gustave Eiffel, UPEC, F-77454, Marne-la-Vallée, France

${ }^{2}$ INRIA Grand-Est, F-67000 Strasbourg, France

${ }^{3}$ IRMA, CNRS, Université de Strasbourg, F-67000 Strasbourg, France

\begin{abstract}
We introduce a semi-implicit two-speed relaxation scheme to solve the compressible Euler equations in the low Mach regime. The scheme involves a relaxation system with two speeds, already introduced by Bouchut, Chalons, Guisset (2019) in the barotropic case. It is entropy satisfying and has a numerical viscosity well-adapted to low Mach flows. This relaxation system is solved via a dynamical Mach number dependent splitting, similar to the one proposed by Iampietro et al. (2018). Stability conditions are derived, they limit the range of admissible relaxation and splitting parameters. We resolve separately the advection part of the splitting by an explicit method, and the acoustic part by an implicit method. The relaxation speeds are chosen so that the implicit system fully linearizes the acoustics and requires just to invert an elliptic operator with constant coefficients. The scheme is shown to well capture with low cost the incompressible slow scale dynamics with a timestep adapted to the velocity field scale, and rather well the fast acoustic waves.
\end{abstract}

Keywords. Compressible Euler equations, Low Mach flows, semi-implicit methods, twospeed relaxation, dynamical splitting, low cost solvers, large timestep

AMS Classification NUMBERs. 76M12, 76M45

\section{Contents}

1 Introduction 2

2 Two relaxation methods for low-Mach flows 5

2.1 Implicit Jin-Xin relaxation scheme . . . . . . . . . . . . . . . . . 6

2.2 Semi-implicit Suliciu relaxation system . . . . . . . . . . . . . 6 
3 A two speed relaxation model for the full Euler system $\quad 8$

3.1 The relaxation model . . . . . . . . . . . . . . . . . . 8

3.2 The asymptotic equivalent equation . . . . . . . . . . . . . . 10

3.3 Stability of the diffusion approximation $\ldots \ldots \ldots \ldots \ldots$

3.4 Entropy stability . . . . . . . . . . . . . . . . . . . . . . . . . . 14

4 Time and space discretization $\quad 16$

4.1 Dynamical splitting . . . . . . . . . . . . . . . . . . . . . . 17

4.2 Stability conditions for the splitting algorithm, and choice of $a, b, \chi_{c}, \chi_{a} \ldots \ldots$

4.3 Numerical schemes for each substep of the splitting . . . . . . . . . . . . . 21

4.3.1 Numerical scheme for the convection step $(C) \ldots \ldots \ldots \ldots$

4.3.2 Numerical scheme for the acoustic step $(A) \ldots \ldots \ldots 22$

4.3.3 Numerical scheme for the relaxation $\operatorname{step}(R) \ldots \ldots \ldots 23$

5 The scheme in several dimensions $\quad 24$

6 Numerical results $\quad 26$

6.1 Contact waves $(1 \mathrm{~d}) \ldots \ldots \ldots \ldots \ldots \ldots$

6.2 Acoustic waves $(1 \mathrm{~d}) \ldots \ldots \ldots \ldots \ldots$

6.3 Shock tube test-case $(1 \mathrm{~d}) \ldots \ldots \ldots \ldots \ldots \ldots \ldots$

6.4 Smooth contact wave $(2 \mathrm{~d}) \ldots \ldots \ldots \ldots \ldots \ldots \ldots$

6.5 Gresho vortex $(2 \mathrm{~d}) \ldots \ldots \ldots \ldots$. . . . . . . . . . . . . . . . . . 39

6.6 Kelvin-Helmholtz instability $(2 \mathrm{~d}) \ldots \ldots \ldots \ldots$. . . . . . . . . . . 41

7 Conclusion $\quad \mathbf{4 2}$

A Scheme based on the Suliciu relaxation system $\quad \mathbf{4 4}$

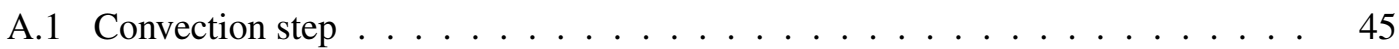

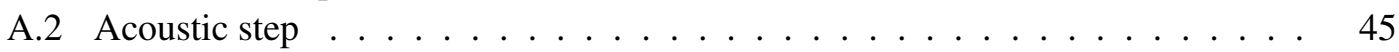

A.3 Relaxation step . . . . . . . . . . . . . . . . . . . 46

B Numerical fluxes for the convection step of the two speed relaxation scheme 46

B.1 Local Lax Friedrichs flux . . . . . . . . . . . . . . . . . . . . . 46

B.2 Linear acoustic flux splitting (FVS) . . . . . . . . . . . . . . 46

B.3 Godunov scheme . . . . . . . . . . . . . . . . . . . 48

\section{Introduction}

Solutions to the compressible Euler system of gas dynamics involve waves that propagate either at material speed or at sound speed. These can be very different in terms of order of magnitude, in particular in the so called low Mach regime when the sound speed is large with respect to the material velocity. In this situation and in order to be efficient, numerical schemes should work with the CFL condition associated with the material speed. It implies that an implicit treatment 
has to be performed on the sound waves, and this should be done with limited loss of accuracy and with explicit cost.

The full Euler system of fluid dynamics can be written

$$
\left\{\begin{array}{l}
\partial_{t} \rho+\nabla \cdot(\rho \boldsymbol{u})=0 \\
\partial_{t}(\rho \boldsymbol{u})+\nabla \cdot(\rho \boldsymbol{u} \otimes \boldsymbol{u}+p \mathrm{Id})=0 \\
\partial_{t} E+\nabla \cdot(E \boldsymbol{u}+p \boldsymbol{u})=0
\end{array}\right.
$$

where $\rho(t, x)$ is the mass density, $\boldsymbol{u}(t, x)$ the fluid velocity, $p(t, x)$ the pressure and $E(t, x)$ the total energy related to the specific internal energy $e(t, x)$ by

$$
E=\rho \frac{|\boldsymbol{u}|^{2}}{2}+\rho e
$$

The system is closed by a state law that relates the pressure $p$ to the density $\rho$ and the internal energy $e$. Additional state variables such as the temperature $T$ and the specific entropy $s$ are attached to the system, but then can be seen as nonlinear functions of $\rho$ and $e$, satisfying the differential relation $d e=\frac{p}{\rho^{2}} d \rho+T d s$.

The low Mach regime is characterized by the property that at density and temperature given, the state law, given under the form $F(\rho, T, p)=0$, does not depend much on the pressure $(\partial F / \partial p$ is small), see [21]. In this formulation the pressure has to be measured at the scale of velocities $\boldsymbol{u}$. The more standard way to describe this regime is to say that the acoustic waves travel much faster than the flow, i.e.

$$
c>|\boldsymbol{u}|,
$$

where the sound speed $c$ is given by $c^{2}=\left(\frac{\partial p}{\partial \rho}\right)_{s}$. Yet another way to formulate the low Mach regime is to say that there is a small time scale attached to the acoustic waves, and a larger time scale attached to the flow. The acoustic waves are anyway of small amplitude compared to the main flow. It we only look for the flow evolution, thus only considering a timestep obeying the CFL condition of the type $\Delta t|\boldsymbol{u}| \leq \Delta x$, it is classical to filter out the acoustic waves, leading to incompressible models.

We would like here to design a numerical method that is able to treat all the regimes (low Mach or not), and which is accurate in the low Mach regime. In particular this means to well resolve the flow scale (close to incompressible), but also to describe as much as possible the acoustic waves. Under the assumption (3) and rescaling the equations so as to have velocities of order unity, we classically have that $\rho, \boldsymbol{u}, T$ remain bounded. For times $t$ at the flow scale a leading expansion is then

$$
\rho=\rho_{0}+O\left(M^{2}\right), \quad \boldsymbol{u}=\boldsymbol{u}_{0}+O\left(M^{2}\right), \quad T=T_{0}+O\left(M^{2}\right),
$$

with $M$ the Mach number i.e. the order of magnitude of $|\boldsymbol{u}| / c$, whereas $\rho_{0}, \boldsymbol{u}_{0}, T_{0}$ represent the leading incompressible flow. Indeed the low Mach assumption (3) enables to write according to [21] that the pressure law can be written

$$
p(\rho, T)=p_{r e f}+p_{s c}(\rho, T) / M^{2},
$$


with $p_{\text {ref }}$ a constant and $p_{s c}$ a scaled pressure law. The state differential relation can then be written

$$
e=-p_{r e f} / \rho+e_{s c} / M^{2}, \quad s=s_{s c} / M^{2}, \quad \text { with } d e_{s c}=\frac{p_{s c}}{\rho^{2}} d \rho+T d s_{s c} .
$$

Then the leading flow is characterized by the incompressible Euler system

$$
\left\{\begin{array}{l}
\partial_{t} \rho_{0}+\boldsymbol{u}_{0} \cdot \nabla \rho_{0}=0 \\
\rho_{0}\left(\partial_{t} \boldsymbol{u}_{0}+\boldsymbol{u}_{0} \cdot \nabla \boldsymbol{u}_{0}\right)+\nabla p_{0}=0 \\
\nabla \cdot \boldsymbol{u}_{0}=0
\end{array}\right.
$$

with $p_{0}$ a Lagrange multiplier and $T_{0}$ is related to $\rho_{0}$ by the constraint $p_{s c}\left(\rho_{0}, T_{0}\right)=0$. Indeed one has

$$
p=p_{0}+O\left(M^{2}\right)
$$

and the incompressibility condition $\nabla \cdot \boldsymbol{u}_{0}=0$ follows from the equation on $p$ that can be deduced from (1), (2),

$$
\partial_{t} p+\boldsymbol{u} \cdot \nabla p+c^{2} \rho \nabla \cdot \boldsymbol{u}=0 .
$$

Coming back to the the original system (1), (2), we can formulate it by including the scaled law (5), as

$$
\left\{\begin{array}{l}
\partial_{t} \rho+\nabla \cdot(\rho \boldsymbol{u})=0, \\
\partial_{t}(\rho \boldsymbol{u})+\nabla \cdot\left(\rho \boldsymbol{u} \otimes \boldsymbol{u}+\frac{p_{s c}}{M^{2}} \mathrm{Id}\right)=0, \\
\partial_{t} E+\nabla \cdot\left(E \boldsymbol{u}+\left(p_{r e f}+\frac{p_{s c}}{M^{2}}\right) \boldsymbol{u}\right)=0
\end{array}\right.
$$

with $E$ related to $e$ by (2), and with (6). In this formulation the $O\left(M^{2}\right)$ corrections of (4) are included, they describe the residual of acoustic waves. We have to mention that at the acoustic time scale, the acoustic waves are perturbations as in (4) but with $O(M)$ instead of $O\left(M^{2}\right)$. They are generated from so called "not well prepared" initial data satisfying $\nabla \cdot \boldsymbol{u}=O(M)$, $p_{s c}(\rho, T)=O(M)\left(O\left(M^{2}\right)\right.$ corresponds to "well prepared" initial data leading to small acoustic waves even at the acoustic time scale). Nevertheless even for "not well prepared" initial data, for times at the flow scale or larger the $O(M)$ part of the acoustic waves have gone (unless iterately reflected by boundaries) and we recover "well prepared" states.

To capture the low Mach regime implies to capture the slow dynamics (7) which is the transport at the fluid velocity of density and velocity, as well as the divergence free constrain. In order to achieve this with a reasonable CPU cost, the time marching scheme is important. Indeed, if we use a standard explicit scheme, we have a CFL condition for the model (1) constrained by the sound speed, which is very large compared to the fluid velocity. This is nevertheless possible since in this case there is no linear system to solve [16]. An alternative method is to use a fully implicit scheme, which leads to a nonlinear problem to invert, with ill-conditioned linear part, because the ratio between the acoustic and physical speeds is large. Another possibility is to use a semi-implicit scheme as in $[27,26,20,18]$ where only the acoustic part is implicit. In this case we obtain a linear problem to invert. This is the method that we adopt here, and we shall pay a particularly attention to the kind of linear system we have to solve. Our aim is to preserve the low Mach limit with good accuracy, and at the same time to have a simple implicit step, as cheap as possible in terms of cpu time and memory. This is obtained via a particular relaxation approach introduced in [14] that has an asymptotic viscosity expressed in terms of $\nabla \cdot \boldsymbol{u}$ and $\nabla p$. 
This is appropriate in the compressible regime in order to well resolve contact discontinuities, across which $\boldsymbol{u} \cdot n$ and $p$ do not jump; and this is also very appropriate in the low Mach regime since $\boldsymbol{u}$ and $p$ remain bounded. It also allows a not too bad resolution of acoustic waves.

In the remainder of the paper we consider the system (1) with (2), keeping in mind the scaling laws (5) and (6) (after an eventual rescaling so that velocity is of order unity), where we take $p_{\text {ref }}=0$. An important example of state law is the stiffened gas law

$$
p=(\gamma-1) \rho e-\gamma p_{\infty}
$$

where $\gamma>1, p_{\infty} \geq 0$ are constants. Then the temperature is given by $c_{p} T / \gamma=e-p_{\infty} / \rho$, where $c_{p}>0$ is a constant, and admissible states are those for which $T>0$. The entropy is given by $s=\frac{c_{p}}{\gamma} \log \left(T / \rho^{\gamma-1}\right)$. The scaling assumptions (5) and (6) are then satisfied if $p_{\infty}=p_{\infty s c} / M^{2}$, $c_{p}=c_{p, s c} / M^{2}$, with $p_{\infty s c}, c_{p, s c}$ constants independent of $M$. The scaled state law $p_{s c}, e_{s c}$ is then a stiffened gas with the constants $p_{\infty s c}, c_{p, s c}$ instead of $p_{\infty}, c_{p}$. In the incompressible regime the relation $p_{s c}(\rho, T)=0$ gives $\rho T=\frac{\gamma}{\gamma-1} \frac{p_{\infty s c}}{c_{p, s c}}=c s t$. We remark that for a perfect gas law $p_{\infty}=0$, the temperature tends to 0 in the incompressible regime. We will first restrict ourselves to the one dimensional case as it already contains an important part of the difficulty. Then in the incompressible regime and with compatible boundary conditions, (7) implies that the fluid velocity $u_{0}$ and the pressure $p_{0}$ are constant. However, contrary to isotropic or barotropic flows, the density $\rho_{0}$ is still inhomogeneous and is advected at velocity $u_{0}$. Hence the one-dimensional low-Mach regime is non trivial in that case. Numerical schemes have to capture the advection of the density, and the emission of acoustic waves for small times of order $M$. The outline of the paper is the following. In Section 2 we briefly present two classes of methods already proposed in the literature for low Mach flows and discuss their advantages and drawbacks in the onedimensional case. In Section 3 we introduce our two-speed relaxation method following [14], and discuss its stability properties. In Section 4 we introduce our numerical scheme based on a particular splitting strategy that allows a low cost linear system to solve. Finally in Section 6 we perform several one and two-dimensional test cases.

\section{Two relaxation methods for low-Mach flows}

In this section we recall two strategies based on relaxation to design implicit/semi-implicit scheme for low Mach flow and we introduce the main issues that we have to tackle: the control of the numerical diffusion error and the numerical cost of the implicit linear solver. 


\subsection{Implicit Jin-Xin relaxation scheme}

The Jin-Xin relaxation model [28] applied to the 1d Euler system consists in considering the extended system

$$
\left\{\begin{aligned}
\partial_{t} \rho+\partial_{x} v_{\rho} & =0, \\
\partial_{t}(\rho u)+\partial_{x} v_{\rho u} & =0, \\
\partial_{t} E+\partial_{x} v_{E} & =0, \\
\partial_{t} v_{\rho}+\lambda^{2} \partial_{x} \rho & =\frac{1}{\varepsilon}\left(\rho u-v_{\rho}\right), \\
\partial_{t} v_{\rho u}+\lambda^{2} \partial_{x}(\rho u) & =\frac{1}{\varepsilon}\left(\rho u^{2}+p-v_{\rho u}\right), \\
\partial_{t} v_{E}+\lambda^{2} \partial_{x} E & =\frac{1}{\varepsilon}\left(E u+p u-v_{E}\right),
\end{aligned}\right.
$$

where $v_{\rho}(t, x), v_{\rho u}(t, x)$ and $v_{E}(t, x)$ are supplementary unknowns and $\lambda>0$ is the relaxation speed. The system (12) has the following interesting features: the left-hand side is a linear hyperbolic system, while all the nonlinearity is in the right-hand side, which is local in space. This system is a stable approximation of the original system under the so-called subcharacteristic condition $\lambda>|u|+c$ with $c$ the acoustic speed.

In [5] an implicit method based on the system (12) was proposed. It consists in using a time splitting scheme between transport and relaxation and to use implicit schemes for each step. The main advantage of this algorithm is that the implicit transport part requires only the inversion of constant Laplacian operators and the computation of matrix-vector products. The limitation of this method lies in the diffusion error. Indeed, the diffusion matrix associated with the relaxation process is given by $\lambda^{2} I_{d}-|A(\rho, u, E)|^{2}$ with $A(\rho, u, E)$ the Jacobian matrix of the Euler flux function, which has eigenvalues $u, u-c, u+c$. Since $\lambda>|u|+c$, the material wave suffers from an error of the order of $c^{2}$. In particular, in the asymptotic incompressible regime, an expansion of the relaxation system gives

$$
\partial_{t} \rho+u \partial_{x} \rho=\Delta t \lambda^{2} \partial_{x x} \rho+O\left(\Delta t^{2}\right) .
$$

Consequently, unless the timestep is taken very small, the diffusion error is large compared with the convection term. It would be desirable to have an error of order $O\left(|u|^{2}\right)$ instead of $O\left(c^{2}\right)$.

\subsection{Semi-implicit Suliciu relaxation system}

Implicit schemes have been designed from the Suliciu relaxation method, [3, 2]. The Suliciu relaxation system [12] writes

$$
\left\{\begin{aligned}
\partial_{t} \rho+\partial_{x}(\rho u) & =0, \\
\partial_{t}(\rho u)+\partial_{x}\left(\rho u^{2}+\Pi\right) & =0, \\
\partial_{t} E+\partial_{x}(E u+\Pi u) & =0, \\
\partial_{t}(\rho \Pi)+\partial_{x}(\rho \Pi u)+\lambda^{2} \partial_{x} u & =\frac{\rho}{\varepsilon}(p-\Pi),
\end{aligned}\right.
$$


where $\Pi(t, x)$ is an auxiliary pressure and $\lambda>0$ is again a relaxation speed. The left-hand side system is hyperbolic with all fields linearly degenerate. The stability of the approximation is ensured by the subcharacteristic condition $\lambda \geq \rho c$.

A semi-implicit scheme can be designed from the Suliciu relaxation method. In [6] the authors introduce a dynamic splitting of this relaxation system between a convective part

$$
\left\{\begin{array}{l}
\partial_{t} \rho+\partial_{x}(\rho u)=0, \\
\partial_{t}(\rho u)+\partial_{x}\left(\rho u^{2}+\mathcal{E}^{2}(t) \Pi\right)=0, \\
\partial_{t} E+\partial_{x}\left(E u+\mathcal{E}^{2}(t) \Pi u\right)=0, \\
\partial_{t}(\rho \Pi)+\partial_{x}(\rho \Pi u)+\lambda_{c}^{2} \partial_{x} u=0,
\end{array}\right.
$$

an acoustic part

$$
\left\{\begin{array}{l}
\partial_{t} \rho=0, \\
\partial_{t}(\rho u)+\left(1-\mathcal{E}^{2}(t)\right) \partial_{x} \Pi=0, \\
\partial_{t} E+\left(1-\mathcal{E}^{2}(t)\right) \partial_{x}(\Pi u)=0, \\
\partial_{t}(\rho \Pi)+\left(1-\mathcal{E}^{2}(t)\right) \lambda_{a}^{2} \partial_{x} u=0,
\end{array}\right.
$$

and a relaxation part

$$
\left\{\begin{aligned}
\partial_{t} \rho & =0, \\
\partial_{t}(\rho u) & =0, \\
\partial_{t} E & =0, \\
\partial_{t}(\rho \Pi) & =\frac{\rho}{\varepsilon}(p-\Pi),
\end{aligned}\right.
$$

where the splitting parameter is defined as

$$
\mathcal{E}(t)=\max \left(M_{\min }, \min \left(M_{\mathrm{eff}}(t), 1\right)\right) \quad \in\left[M_{\min }, 1\right],
$$

with the effective Mach number defined as

$$
M_{\mathrm{eff}}(t)=\sup _{x} \frac{|u(t, x)|}{c(t, x)} .
$$

The lower bound $M_{\min }>0$ imposed on $\mathcal{E}(t)$ guarantees (14) to remain hyperbolic, it is needed if we have data for which $u \equiv 0$.

We have the free parameters $\lambda_{c}>0, \lambda_{a}>0$ in the splitting of the auxiliary pressure equation, that have to satisfy $\lambda^{2}=\lambda_{c}^{2}+\left(1-\mathcal{E}^{2}(t)\right) \lambda_{a}^{2}$. Note that in [6] the scheme is constructed in the opposite order, by first introducing the splitting and then relaxation systems. The splitting has the property to separate the multi-scale dynamics. All the waves of the convective part (14) have $O(u)$ speeds while the acoustic part (15) have $O(c)$ waves speeds. Hence a classical explicit finite volume scheme can be used for the convective part and generates only $O(u)$ diffusion terms. For the acoustic system (15), a classical strategy is to use a parabolization method $[5,22,4]$, thus 
considering the time implicit scheme

$$
\left\{\begin{aligned}
\rho^{n} u^{n+1} & =\rho^{n} u^{n}-\Delta t\left(1-\mathcal{E}^{2}(t)\right) \partial_{x} \Pi^{n+1}, \\
E^{n+1} & =E^{n}-\Delta t\left(1-\mathcal{E}^{2}(t)\right) \partial_{x}\left(\Pi^{n+1} u^{n+1}\right), \\
\rho^{n} \Pi^{n+1} & =\rho^{n} \Pi^{n}-\Delta t\left(1-\mathcal{E}^{2}(t)\right) \lambda_{a}^{2} \partial_{x} u^{n+1} .
\end{aligned}\right.
$$

Plugging the third equation into the first one, we obtain the elliptic equation

$$
u^{n+1}-\Delta t^{2}\left(1-\mathcal{E}^{2}(t)\right)^{2} \frac{1}{\rho^{n}} \partial_{x}\left(\frac{\lambda_{a}^{2}}{\rho^{n}} \partial_{x} u^{n+1}\right)=u^{n}-\Delta t\left(1-\mathcal{E}^{2}(t)\right) \frac{1}{\rho^{n}} \partial_{x} \Pi^{n} .
$$

When this elliptic equation is solved we get $u^{n+1}$, and then we can compute $\Pi^{n+1}$ and $E^{n+1}$ from (19). Contrary to the implicit Jin -Xin relaxation scheme presented in the previous section, here we need to invert a non homogeneous Laplacian operator. This requires to assemble a new matrix at each timestep, which could have an important CPU cost for 2D or 3D simulations. Moreover the matrix may have a large condition number when the density has large spatial variations.

This whole scheme is detailed in appendix A. Note that another strategy has been proposed in $[3,2]$ for the implicit treatment of the acoustic system. It is based on the diagonalization of the $(\Pi, u)$ subsystem and the resolution of the two resulting transport equations with an implicit solver. However, we face the same difficulties: the transport equations are non-homogeneous and thus also require a matrix assembly at each timestep.

The conclusion of this section is that the first method allows to obtain very simple implicit steps, but admits a very large error in the low-Mach regime. The second method is accurate in the low-Mach regime but requires assembling a new matrix at each timestep. In the following we propose a scheme that combines the advantages of the two previous methods.

\section{A two speed relaxation model for the full Euler system}

In this section we introduce a two-speed relaxation model for the full Euler system (1), following the strategy considered in [14] for the barotropic case.

\subsection{The relaxation model}

The two speed relaxation system for the full Euler system (1) in one dimension writes

$$
\left\{\begin{array}{l}
\partial_{t} \rho+\partial_{x}(\rho v)=0, \\
\partial_{t}(\rho u)+\partial_{x}(\rho u v)+\partial_{x} \Pi=0, \\
\partial_{t} E+\partial_{x}(E v)+\partial_{x}(\Pi v)=0, \\
\partial_{t}(\rho \Pi)+\partial_{x}(\rho \Pi v)+a b \partial_{x} v=\frac{\rho}{\varepsilon}(p-\Pi), \\
\partial_{t}(\rho v)+\partial_{x}\left(\rho v^{2}\right)+\frac{a}{b} \partial_{x} \Pi=\frac{\rho}{\varepsilon}(u-v),
\end{array}\right.
$$


where still $E=\rho u^{2} / 2+\rho e$ and $p \equiv p(\rho, e)$ is the state law. In this system, $\Pi(t, x)$ and $v(t, x)$ are relaxation variables that approximate $p$ and $u$ respectively, and $a(t, x)>0, b(t, x)>0$ are auxiliary positive speed relaxation variables that are advected by the flow

$$
\left\{\begin{array}{l}
\partial_{t}(\rho a)+\partial_{x}(\rho a v)=0 \\
\partial_{t}(\rho b)+\partial_{x}(\rho b v)=0
\end{array}\right.
$$

This system is the direct extension of the relaxation model proposed in [14] for the barotropic case. Notice that when $a \equiv b$, one has $v \equiv u$ and we recover the usual Suliciu relaxation model (13) with $a=b=\lambda$. The parameters $a, b$ need to respect some stability inequalities, as shown below.

This relaxation method has the advantage to have two relaxation variables that follow an approximate acoustic dynamics. But as auxiliary variables we can make their propagation speed constant by choosing adequately the speeds $a$ and $b$. Therefore it will be possible to define a semi-implicit discretization with homogeneous elliptic equation as we will see in Section 4.

We notice that the system (20), (21) can be written under the following nonconservative form

$$
\left\{\begin{array}{l}
\partial_{t} \rho+\partial_{x}(\rho v)=0, \\
\partial_{t}(\rho u)+\partial_{x}(\rho u v)+\partial_{x} \Pi=0, \\
\partial_{t} E+\partial_{x}(E v)+\partial_{x}(\Pi v)=0, \\
\partial_{t} \Pi+v \partial_{x} \Pi+\frac{a b}{\rho} \partial_{x} v=\frac{1}{\varepsilon}(p-\Pi), \\
\partial_{t} v+v \partial_{x} v+\frac{a}{b \rho} \partial_{x} \Pi=\frac{1}{\varepsilon}(u-v), \\
\partial_{t} a+v \partial_{x} a=0, \\
\partial_{t} b+v \partial_{x} b=0 .
\end{array}\right.
$$

Then, the following lemma characterizes the hyperbolicity of the relaxation system.

Lemma 3.1. The relaxation system (20), (21) is hyperbolic and all the characteristic fields are linearly degenerate. The characteristic speeds are given by:

$$
\Sigma=\left\{v, v, v, v, v, v-\frac{a}{\rho}, v+\frac{a}{\rho}\right\} .
$$

Proof. The computation of the eigenvalues of the Jacobian matrix of the flux is easy and left to the reader. The eigenvalues can also be deduced from the following argument: the relaxation system (20) is the same as that in [14] except for the addition of a new unknown $E$, or equivalently $e$ or $\widehat{e}$, that satisfies (41), (42). We conclude that as in [14] the system is hyperbolic with all eigenvalues linearly degenerate. The solution to the Riemann problem is also the same as in [14].

We notice that if $a \simeq \rho c$ and $b \simeq \rho c$, we obtain that the extreme eigenvalues of (23) verify $v \pm \frac{a}{\rho} \simeq u \pm c$, the eigenvalues of the original Euler system (1). On the contrary, in order to be accurate in the low Mach limit, in [14] the authors propose to choose $a \simeq \rho c / \min (1, M)$ with 
$M$ the Mach number (which means that $|u| / c$ is of the order of $M$ ) and $b \simeq \rho c \min (1, M)$. This choice satisfies the subcharacteristic conditions (30). Then the eigenvalues (23) of the relaxation system are much larger than those of the Euler system, but $b$ remains bounded for small $M$, a condition that guarantees to keep a finite numerical viscosity. This choice for $a$ and $b$, when discretizing in space [14] and applying an explicit treatment, leads to a numerical viscosity of the form (25) where we replace formally in the case of small $M$

$$
\varepsilon\left(\frac{a}{b}-1\right) \longrightarrow \frac{\Delta x}{2 c M}, \quad \varepsilon\left(\frac{a b}{\rho^{2}}-c^{2}\right) \longrightarrow \frac{\Delta x}{2}(|u|+c M) .
$$

This implies that the scheme is then first order uniformly for small $M$. However this approach induces a very restrictive CFL condition $\Delta t a / \rho \leq \Delta x$ since $a$ is large. Moreover it is not possible to bypass this difficulty with an implicit treatment, because for timesteps $\Delta t$ significantly larger than $\rho \Delta x / a$ the time diffusion will be large, see Lemma 3.2 below where $\varepsilon$ has to be understood as $\Delta t / 2$ according to [13].

In the present paper we introduce a semi-implicit scheme with a different choice for $a$ and $b$, that enables to have a moderate diffusion in time for large timesteps.

\subsection{The asymptotic equivalent equation}

The asymptotic diffusion in the relaxation parameter $\varepsilon$, that is related to time, depends on the choice of $a$ and $b$. It is given by the following formulas.

Lemma 3.2. As $\varepsilon \rightarrow 0$ the relaxation system (20)-(21) provides the following first order in $\varepsilon$ approximation of the Euler system

$$
\left\{\begin{aligned}
\partial_{t} \rho+\partial_{x}(\rho u) & =\varepsilon \partial_{x}\left(\delta_{1} \partial_{x} p\right), \\
\partial_{t}(\rho u)+\partial_{x}\left(\rho u^{2}+p\right) & =\varepsilon \partial_{x}\left(\delta_{1} u \partial_{x} p+\delta_{2} \partial_{x} u\right), \\
\partial_{t} E+\partial_{x}(E u+p u) & =\varepsilon \partial_{x}\left(\delta_{1} \frac{E}{\rho} \partial_{x} p+\delta_{1} \frac{p}{\rho} \partial_{x} p+\delta_{2} u \partial_{x} u\right),
\end{aligned}\right.
$$

with $\delta_{1}=\frac{a}{b}-1$ and $\delta_{2}=\frac{a b-\rho^{2} c^{2}}{\rho}$.

We notice that the relaxation system is an approximation of the Euler system with diffusion fluxes all depending linearly on $\partial_{x} u$ and $\partial_{x} p$. This is the key property for capturing contact discontinuities since $u$ and $p$ do not jump through these, as well as the low-Mach regime since in the latter case $u$ and $p$ remain bounded, as explained in the introduction.

Proof. We perform an Hilbert expansion with respect to powers of $\varepsilon$ for all the unknowns: $\rho=\rho_{0}+\varepsilon \rho_{1}+O\left(\varepsilon^{2}\right), u=u_{0}+\varepsilon u_{1}+O\left(\varepsilon^{2}\right)$, etc. Then we plug these expansions into (22) and identify terms of equal powers of $\varepsilon$.

The terms of order $1 / \varepsilon$ lead to

$$
\Pi_{0}=p_{0}, \quad v_{0}=u_{0} .
$$


The terms of order 1 in the first three equations of (22) satisfy the system

$$
\left\{\begin{array}{l}
\partial_{t} \rho_{0}+\partial_{x}\left(\rho_{0} v_{0}\right)=0 \\
\partial_{t}\left(\rho_{0} u_{0}\right)+\partial_{x}\left(\rho_{0} u_{0} v_{0}+\Pi_{0}\right)=0, \\
\partial_{t} E_{0}+\partial_{x}\left(E_{0} v_{0}+v_{0} \Pi_{0}\right)=0 .
\end{array}\right.
$$

According to (26), this is equivalent to

$$
\left\{\begin{array}{l}
\partial_{t} \rho_{0}+\partial_{x}\left(\rho_{0} u_{0}\right)=0 \\
\partial_{t}\left(\rho_{0} u_{0}\right)+\partial_{x}\left(\rho_{0} u_{0}^{2}+p_{0}\right)=0 \\
\partial_{t} E_{0}+\partial_{x}\left(E_{0} u_{0}+p_{0} u_{0}\right)=0
\end{array}\right.
$$

The equation on $\Pi$ leads to an expression for $\Pi_{1}$

$$
\begin{aligned}
\Pi_{1} & =p_{1}-\left(v_{0} \partial_{x} \Pi_{0}+\frac{a b}{\rho_{0}} \partial_{x} v_{0}+\partial_{t} p_{0}\right) \\
& =p_{1}-\left(u_{0} \partial_{x} p_{0}+\frac{a b}{\rho_{0}} \partial_{x} u_{0}-u_{0} \partial_{x} p_{0}-c_{0}^{2} \rho_{0} \partial_{x} u_{0}\right) \\
& =p_{1}-\frac{1}{\rho_{0}}\left(a b-\rho_{0}^{2} c_{0}^{2}\right) \partial_{x} u_{0},
\end{aligned}
$$

where we used the relations (26) and where $c$ stands for the sound speed, $c^{2}=\left(\frac{\partial p}{\partial \rho}\right)_{s}$. Similarly from the equation on $v$ we get the an expression for $v_{1}$

$$
\begin{aligned}
v_{1} & =u_{1}-\left(v_{0} \partial_{x} v_{0}+\frac{a}{b \rho_{0}} \partial_{x} \Pi_{0}+\partial_{t} u_{0}\right) \\
& =u_{1}-\left(u_{0} \partial_{x} u_{0}+\frac{a}{b \rho_{0}} \partial_{x} p_{0}-u_{0} \partial_{x} u_{0}-\frac{1}{\rho_{0}} \partial_{x} p_{0}\right) \\
& =u_{1}-\frac{1}{\rho_{0}}\left(\frac{a}{b}-1\right) \partial_{x} p_{0} .
\end{aligned}
$$

We thus have

$$
\Pi_{1}=p_{1}-\delta_{2} \partial_{x} u_{0}, \quad v_{1}=u_{1}-\frac{\delta_{1}}{\rho_{0}} \partial_{x} p_{0},
$$

with the above definitions of $\delta_{1}$ and $\delta_{2}$.

Finally the terms in $\varepsilon$ lead to the system

$$
\left\{\begin{array}{l}
\partial_{t} \rho_{1}+\partial_{x}(\rho v)_{1}=0, \\
\partial_{t}(\rho u)_{1}+\partial_{x}(\rho u v)_{1}+\partial_{x} \Pi_{1}=0, \\
\partial_{t} E_{1}+\partial_{x}(E v)_{1}+\partial_{x}(\Pi v)_{1}=0,
\end{array}\right.
$$

where $(f g)_{1}$ stands for $f_{0} g_{1}+f_{0} g_{1}$ for any quantities $f, g$. Using (26) and (28) we get

$$
\begin{aligned}
(\Pi v)_{1}=\Pi_{1} v_{0}+\Pi_{0} v_{1} & =u_{0} p_{1}+p_{0} u_{1}-u_{0} \delta_{2} \partial_{x} u_{0}-p_{0} \frac{\delta_{1}}{\rho_{0}} \partial_{x} p_{0} \\
& =(p u)_{1}-u_{0} \delta_{2} \partial_{x} u_{0}-p_{0} \frac{\delta_{1}}{\rho_{0}} \partial_{x} p_{0} .
\end{aligned}
$$


Similarly we have for the convective fluxes

$$
\begin{aligned}
(\rho v)_{1} & =\rho_{0} v_{1}+\rho_{1} v_{0}=(\rho u)_{1}-\delta_{1} \partial_{x} p_{0}, \\
(\rho u v)_{1} & =(\rho u)_{0} v_{1}+(\rho u)_{1} v_{0}=\left(\rho u^{2}\right)_{1}-u_{0} \delta_{1} \partial_{x} p_{0}, \\
(E v)_{1} & =E_{0} v_{1}+E_{1} v_{0}=(E u)_{1}-\frac{E_{0}}{\rho_{0}} \delta_{1} \partial_{x} p_{0} .
\end{aligned}
$$

Plugging these expressions in (29) and then adding systems (27) and $\varepsilon$ times (29), we obtain the system satisfied by $\rho_{0}+\varepsilon \rho_{1},(\rho u)_{0}+\varepsilon(\rho u)_{1}$ and $E_{0}+\varepsilon E_{1}$, which is the system (25).

\subsection{Stability of the diffusion approximation}

A first step in proving stability of the relaxation system is to evaluate the dissipation of the diffusive approximation [13]. This provides stability conditions on the parameters of the problem.

Lemma 3.3. The diffusive system (25) is entropically stable if and only if

$$
\frac{a b}{\rho^{2}} \geq c^{2} \quad \text { and } \quad a \geq b .
$$

Proof. The Euler system (1) has an entropy

$$
\eta=\rho \phi(s),
$$

where $s=s(\rho, e)$ is the specific entropy, related to the density and internal energy by the differential relation $d e=\frac{p}{\rho^{2}} d \rho+T d s$, with $T$ the temperature. The function $\phi$ is a nonlinearity that satisfies $\phi^{\prime} \leq 0$ and $\phi^{\prime \prime} \geq 0$. Under the classical assumption that $-s$ is a convex function of $(1 / \rho, e)$, we have that $\eta$ is a convex function of the conservative variable $(\rho, \rho u, E)$, see [12, Lemma 1.3]. Then for the entropic stability of the diffusive system (25), we have to write that the diffusive part is symmetrically entropy dissipative. According to [13, Definition 2.11], this means that the matrix $D^{t} \eta^{\prime \prime}$ is symmetric nonnegative, where $D$ is the diffusion matrix, and $\eta^{\prime \prime}$ is the second-order derivative of $\eta$ with respect to the conservative variable $U=(\rho, \rho u, E)$. The definition of the diffusion matrix $D$ is that the right-hand side of (25) has to be written in vector form as $\varepsilon \partial_{x}\left(D \partial_{x} U\right)$. Thus according to (25), the diffusion matrix is given in terms of its lines by

$$
D=\left(\begin{array}{c}
\alpha p^{\prime} \\
\alpha u p^{\prime}+\beta u^{\prime} \\
\frac{E+p}{\rho} \alpha p^{\prime}+\beta u u^{\prime}
\end{array}\right)=\left(\begin{array}{c}
1 \\
u \\
\frac{E+p}{\rho}
\end{array}\right) \alpha p^{\prime}+\left(\begin{array}{l}
0 \\
1 \\
u
\end{array}\right) \beta u^{\prime},
$$

with

$$
\alpha=\delta_{1}=\frac{a}{b}-1, \quad \beta=\delta_{2}=\frac{a b-\rho^{2} c^{2}}{\rho} .
$$

Here $p^{\prime}$ and $u^{\prime}$ stand for the derivatives of $p$ and $u$ with respect to $U$. These are linear forms, identified with line vectors. Using the identity $E=\rho u^{2} / 2+\rho e$ we get

$$
d E=\frac{u^{2}}{2} d \rho+\rho u d u+e d \rho+\rho d e, \quad d(\rho u)=u d \rho+\rho d u .
$$


Thus with the thermodynamical identity $d e=\frac{p}{\rho^{2}} d \rho+T d s$ we obtain

$$
\begin{aligned}
s^{\prime} & =\frac{1}{T}\left(e^{\prime}-\frac{p}{\rho^{2}} \rho^{\prime}\right) \\
& =\frac{1}{T}\left(-\frac{e}{\rho}+\frac{u^{2}}{2 \rho}-\frac{p}{\rho^{2}},-\frac{u}{\rho}, \frac{1}{\rho}\right) \\
& =\frac{1}{\rho T}\left(\frac{u^{2}}{2}-e-\frac{p}{\rho},-u, 1\right) .
\end{aligned}
$$

Next we differentiate (31) and get

$$
\eta^{\prime}=\phi(s)(1,0,0)+\frac{\phi^{\prime}(s)}{T}\left(\frac{u^{2}}{2}-(e+p / \rho),-u, 1\right) .
$$

In order to compute $\eta^{\prime \prime}$ we have to differentiate once more the previous formula. We compute

$$
\begin{aligned}
& \left(\left(\frac{u^{2}}{2}-(e+p / \rho),-u, 1\right)^{t}\right)^{\prime} \\
= & \left(\begin{array}{ccc}
-\frac{\partial_{\rho} p}{\rho}-\frac{1}{\rho T}\left(T+\frac{\partial_{s} p}{\rho}\right)\left(\frac{u^{2}}{2}-(e+p / \rho)\right)-\frac{u^{2}}{\rho} & \frac{u}{\rho T}\left(T+\frac{\partial_{s} p}{\rho}\right)+\frac{u}{\rho} & -\frac{1}{\rho T}\left(T+\frac{\partial_{s} p}{\rho}\right) \\
\frac{u}{\rho} & -\frac{1}{\rho} & 0 \\
0 & 0 & 0
\end{array}\right),
\end{aligned}
$$

where $\partial_{\rho} p$ and $\partial_{s} p$ denote the derivatives of $p$ with respect to $\rho$ at $s$ constant and with respect to $s$ at $\rho$ constant, respectively. Thus we obtain

$$
\begin{aligned}
\eta^{\prime \prime}= & \frac{\phi^{\prime}(s)}{T}\left(\begin{array}{ccc}
-\frac{\partial_{\rho} p}{\rho}-\frac{\partial_{s} p}{\rho^{2} T}\left(\frac{u^{2}}{2}-(e+p / \rho)\right)-\frac{u^{2}}{\rho} & u \frac{\partial_{s} p}{\rho^{2} T}+\frac{u}{\rho} & -\frac{\partial_{s} p}{\rho^{2} T} \\
\frac{u}{\rho} & -\frac{1}{\rho} & 0 \\
0 & 0 & 0
\end{array}\right) \\
& +\left(\frac{u^{2}}{2}-(e+p / \rho),-u, 1\right)^{t}\left(\frac{\phi^{\prime}(s)}{T}\right)^{\prime} .
\end{aligned}
$$

We have to write that $D^{t} \eta^{\prime \prime}$ is symmetric nonnegative. Since $\left(\frac{u^{2}}{2}-(e+p / \rho),-u, 1\right)$ is orthogonal to the vectors $\left(1, u, \frac{u^{2}}{2}+e+p / \rho\right)$ and $(0,1, u)$, we notice that in the left product with $D^{t}$ (with $D$ given by (32)), the second line of (35) gives a vanishing contribution. Thus we only have to consider the first line of (35), and we get

$$
\begin{aligned}
D^{t} \eta^{\prime \prime}= & \alpha\left(p^{\prime}\right)^{t} \frac{\phi^{\prime}(s)}{T}\left(-\frac{\partial_{\rho} p}{\rho}-\frac{\partial_{s} p}{\rho^{2} T}\left(\frac{u^{2}}{2}-(e+p / \rho)\right), u \frac{\partial_{s} p}{\rho^{2} T},-\frac{\partial_{s} p}{\rho^{2} T}\right) \\
& +\beta\left(u^{\prime}\right)^{t} \frac{\phi^{\prime}(s)}{T}\left(\frac{u}{\rho},-\frac{1}{\rho}, 0\right) \\
= & -\alpha \frac{\phi^{\prime}(s)}{\rho T}\left(p^{\prime}\right)^{t} p^{\prime}-\beta \frac{\phi^{\prime}(s)}{T}\left(u^{\prime}\right)^{t} u^{\prime} .
\end{aligned}
$$

This matrix is symmetric, and since $p^{\prime}$ and $u^{\prime}$ are independent linear forms, the matrix is nonnegative if and only if $\alpha \geq 0$ and $\beta \geq 0$, which yields (30). 


\subsection{Entropy stability}

In this section we prove that the relaxation system (20) is entropy satisfying, meaning that the physical entropy is necessarily increasing in the reference frame of the flow when starting with an initial condition at equilibrium. Entropy inequalities allow in good cases to prove the convergence of relaxation models [11]. We prove here the increase of entropy at least in the transportprojection case, which means that the relaxation is replaced by a projection onto equilibrium every timestep.

Lemma 3.4. Under the stability inequalities (30), the system (20), (21) is entropy satisfying, in the sense that solving it without right-hand side and taking initial data at equilibrium $(\Pi=$ $p(\rho, e)$ and $v=u)$, one has

$$
s(\rho, e) \geq \widehat{s},
$$

where $\widehat{s}$ is defined as solving

$$
\partial_{t} \widehat{s}+v \partial_{x} \widehat{s}=0,
$$

with initial data at equilibrium $\widehat{s}=s(\rho, e)$. Here, $s(\rho, e)$ stands for the specific entropy related to the state law.

Proof. We recall that the specific entropy $s$ is related to the other internal variables by the relation $d e=\frac{p}{\rho^{2}} d \rho+T d s$, with $T$ the temperature. The system can be written as (22), without the righthand side, and with $E=\rho u^{2} / 2+\rho e$. We subtract the equation of $v$ to the one on $u$ to get

$$
\partial_{t}(v-u)+v \partial_{x}(v-u)+\left(\frac{a}{b}-1\right) \frac{1}{\rho} \partial_{x} \Pi=0 .
$$

We multiply this by $(v-u)$ and obtain

$$
\partial_{t} \frac{(v-u)^{2}}{2}+v \partial_{x} \frac{(v-u)^{2}}{2}+\left(\frac{a}{b}-1\right) \frac{1}{\rho}(v-u) \partial_{x} \Pi=0 .
$$

Then, multiplying by $\Pi$ the $\Pi$ equation we get

$$
\partial_{t} \frac{\Pi^{2}}{2}+v \partial_{x} \frac{\Pi^{2}}{2}+\frac{a b}{\rho} \Pi \partial_{x} v=0
$$

Adding the two previous equations and using that $a$ and $b$ are in the kernel of $\partial_{t}+v \partial_{x}$, we obtain

$$
\partial_{t}\left(\frac{\Pi^{2}}{2 a b}+\frac{(v-u)^{2}}{2\left(\frac{a}{b}-1\right)}\right)+v \partial_{x}\left(\frac{\Pi^{2}}{2 a b}+\frac{(v-u)^{2}}{2\left(\frac{a}{b}-1\right)}\right)+\frac{1}{\rho} \partial_{x}(\Pi v)-\frac{u}{\rho} \partial_{x} \Pi=0
$$

In the previous formula we agree with the convention that $(v-u)^{2} /(a / b-1)$ vanishes in the case when $a=b$, because in this case we have $v \equiv u$. One easily gets the equation on $u^{2}$

$$
\partial_{t} \frac{u^{2}}{2}+v \partial_{x} \frac{u^{2}}{2}+\frac{u}{\rho} \partial_{x} \Pi=0
$$


Next we can write the equation satisfied by $E / \rho$,

$$
\partial_{t} \frac{E}{\rho}+v \partial_{x} \frac{E}{\rho}+\frac{1}{\rho} \partial_{x}(\Pi v)=0,
$$

and since $E / \rho=u^{2} / 2+e$, we obtain

$$
\partial_{t} e+v \partial_{x} e+\frac{1}{\rho} \partial_{x}(\Pi v)-\frac{u}{\rho} \partial_{x} \Pi=0 .
$$

Define now $\widehat{e}$ by

$$
e=\widehat{e}+\frac{(v-u)^{2}}{2\left(\frac{a}{b}-1\right)} \geq \widehat{e},
$$

the inequality holding because of the second stability condition in (30). Subtracting (39) to (40) and using (41) we deduce that

$$
\partial_{t}\left(\widehat{e}-\frac{\Pi^{2}}{2 a b}\right)+v \partial_{x}\left(\widehat{e}-\frac{\Pi^{2}}{2 a b}\right)=0 .
$$

Now we can observe that the relaxation system (20) is the same as that in [14] except for the addition of a new unknown $E$, or equivalently $e$ or $\widehat{e}$, that satisfies (42). We conclude that the system is hyperbolic with all eigenvalues linearly degenerate, and this justifies to use the chain rule in all the computations. Then, using the mass equation we get

$$
\partial_{t} \frac{1}{\rho}+v \partial_{x} \frac{1}{\rho}-\frac{1}{\rho} \partial_{x} v=0,
$$

and combining it with the $\Pi$ equation of (22) we get

$$
\partial_{t}\left(\Pi+\frac{a b}{\rho}\right)+v \partial_{x}\left(\Pi+\frac{a b}{\rho}\right)=0 .
$$

We now introduce a new independent variable $\tau$ solving

$$
\partial_{t} \tau+v \partial_{x} \tau=0,
$$

initialized as $\tau=1 / \rho$. Using this equation and the equation on $\widehat{s}$ in (38) we get $\left(\partial_{t}+v \partial_{x}\right)(p(1 / \tau, \widehat{s})+$ $a b \tau)=0$, where $p(\rho, s)$ stands for the pressure law written as a function of density $\rho$ and specific entropy $s$. Since $\Pi+a b / \rho$ and $p(1 / \tau, \widehat{s})+a b \tau$ take initially the same value and solve the same equation, we deduce that they are equal,

$$
\Pi+a b / \rho=p(1 / \tau, \widehat{s})+a b \tau
$$

With the same argument, we have that

$$
\widehat{e}-\frac{\Pi^{2}}{2 a b}=e(1 / \tau, \widehat{s})-\frac{p(1 / \tau, \widehat{s})^{2}}{2 a b} .
$$


Therefore, eliminating $\pi$ with the last two identities, we obtain an expression of $\widehat{e}$ as

$$
\begin{aligned}
\widehat{e} & =e(1 / \tau, \widehat{s})-\frac{p(1 / \tau, \widehat{s})^{2}}{2 a b}+\frac{(p(1 / \tau, \widehat{s})+a b \tau-a b / \rho)^{2}}{2 a b} \\
& =e(1 / \tau, \widehat{s})+p(1 / \tau, \widehat{s})(\tau-1 / \rho)+\frac{a b}{2}(\tau-1 / \rho)^{2} .
\end{aligned}
$$

We observe then that as proved in [14], this expression is larger than $e(\rho, \widehat{s})$ as soon as

$$
\sup _{\bar{\rho} \in[\rho, 1 / \tau]} \bar{\rho}^{2}\left(\frac{\partial p}{\partial \rho}\right)_{s}(\bar{\rho}, \widehat{s}) \leq a b .
$$

This inequality is thus the precise sense in which we have to understand the first stability condition in (30). Under this condition we conclude that

$$
e \geq \widehat{e} \geq e(\rho, \widehat{s}),
$$

and since $(\partial e / \partial s)_{\rho}=T>0$, we conclude that $s(\rho, e) \geq \widehat{s}$, which is the claim.

Corollary 3.5. Consider the explicit relaxation scheme for (1) obtained by solving the Riemann problem for the relaxation system (20), (21) without right-hand side, with data at equilibrium $\Pi^{L}=p\left(\rho^{L}, e^{L}\right), \Pi^{R}=p\left(\rho^{R}, e^{R}\right), v^{L}=u^{L}, v^{R}=u^{R}$. We assume that $a^{L}, b^{L}, a^{R}, b^{R}$ are such that the intermediate densities are positive and the Riemann solution satisfies the subcharacteristic conditions (30) in the precised sense (45), for all intermediate states. Then the scheme verifies a fully discrete entropy inequality for the entropy $\rho \phi(s)$, for any $\phi$ such that $\phi^{\prime} \leq 0$ and $\phi^{\prime \prime} \geq 0$. Moreover, the scheme satisfies the minimum principle on $s$.

Proof. As in [14] the assumption of positive intermediate densities ensure that the solution to the Riemann problem exists. Because of (38) one has

$$
\partial_{t}(\rho \phi(\widehat{s}))+\partial_{x}(\rho v \phi(\widehat{s}))=0 .
$$

We integrate this equation over a time-space rectangle containing the Riemann fan. Using that the initial data are at equilibrium and the inequality (37) at final time, we obtain the fully discrete entropy inequality. Another way to argue is to notice that with (46), $\rho \phi(\widehat{s})$ and $\rho v \phi(\widehat{s})$ play the role of extended entropy and entropy flux respectively. Then (37) says that $\rho \phi(s) \leq \rho \phi(s)$, and the proof follows the lines of [14, Proposition 1]. Finally, taking $\phi(s)=(k-s)_{+}$for some constant $k$ shows that if $s \geq k$ initially then it remains true for all time, that is the minimum principle on $s$ holds.

We notice that following [14] it is possible to give explicit values for $a^{L}, b^{L}, a^{R}, b^{R}$ so that the intermediate densities are positive and the subcharacteristic conditions are satisfied. However, as commented after Lemma 3.1, we shall not use this explicit scheme here.

\section{Time and space discretization}

We introduce here the discretization of the two-speed relaxation system (20)-(21). We consider a dynamical splitting methodology as previously introduced in [6] and presented in Section 2.2. 


\subsection{Dynamical splitting}

The aim is to separate the slow scale dynamics, that will be solved with an explicit scheme, from the fast scale dynamics that we will be solved with an implicit one. We consider a parameter $\mathcal{E}^{2}(t) \in\left[M_{\min }^{2}, 1\right]$ as defined in (17). In practice, $\mathcal{E}^{2}(t)$ is recalculated at the beginning of each timestep and is constant during the timestep. We consider the splitting between a convection step, denoted by $(C)$ :

$$
\left\{\begin{array}{l}
\partial_{t} \rho+\partial_{x}(\rho v)=0 \\
\partial_{t}(\rho u)+\partial_{x}(\rho u v)+\mathcal{E}^{2}(t) \partial_{x} \Pi=0 \\
\partial_{t} E+\partial_{x}(E v)+\mathcal{E}^{2}(t) \partial_{x}(\Pi v)=0 \\
\partial_{t} \Pi+v \partial_{x} \Pi+\chi_{c} \frac{a b}{\rho} \partial_{x} v=0 \\
\partial_{t} v+v \partial_{x} v+\mathcal{E}^{2}(t) \frac{a}{b \rho} \partial_{x} \Pi=0 \\
\partial_{t} a+v \partial_{x} a=0 \\
\partial_{t} b+v \partial_{x} b=0
\end{array}\right.
$$

an acoustic step, denoted by $(A)$ :

$$
\left\{\begin{array}{l}
\partial_{t} \rho=0 \\
\partial_{t}(\rho u)+\left(1-\mathcal{E}^{2}(t)\right) \partial_{x} \Pi=0 \\
\partial_{t} E+\left(1-\mathcal{E}^{2}(t)\right) \partial_{x}(\Pi v)=0 \\
\partial_{t} \Pi+\left(1-\mathcal{E}^{2}(t)\right) \chi_{a} \frac{a b}{\rho} \partial_{x} v=0 \\
\partial_{t} v+\left(1-\mathcal{E}^{2}(t)\right) \frac{a}{b \rho} \partial_{x} \Pi=0 \\
\partial_{t} a=0 \\
\partial_{t} b=0
\end{array}\right.
$$

and a relaxation step, denoted by $(R)$ :

$$
\left\{\begin{array}{l}
\partial_{t} \rho=0, \quad \partial_{t}(\rho u)=0, \quad \partial_{t} E=0, \\
\partial_{t} \Pi=\frac{1}{\varepsilon}(p-\Pi), \quad \partial_{t} v=\frac{1}{\varepsilon}(u-v), \quad \partial_{t} a=0, \quad \partial_{t} b=0 .
\end{array}\right.
$$

The parameters $\chi_{c}$ and $\chi_{a}$ in the $\Pi$ equations of $(C)$ and $(A)$ are two extra degrees of freedom. In order to be consistent with the $\Pi$ equation of (22) they have to satisfy

$$
\chi_{c}+\left(1-\mathcal{E}^{2}(t)\right) \chi_{a}=1 .
$$

We notice however that the real consistency we need is with (1), and for that the $\Pi$ equation of (22) is not needed. Thus the relation (50) is not strictly necessary.

The above splitting means that in order to solve our relaxation system (22) over one timestep $\Delta t$, we first solve the convective system $(C)$ over a timestep $\Delta t$. Then we solve the system $(A)$ 
during the timestep $\Delta t$, with initial data the final data obtained from the resolution of the first system. Finally, we solve $(R)$ during the timestep $\Delta t$, with initial data the final data obtained from the resolution of the second system.

The eigenstructure of systems $(C)$ and $(A)$ is as follows.

Lemma 4.1. The characteristic speeds of system $(C)$ are given by

$$
\Sigma_{C}=\left\{v, v, v, v, v, v-\mathcal{E}(t) \sqrt{\chi_{c}} \frac{a}{\rho}, v+\mathcal{E}(t) \sqrt{\chi_{c}} \frac{a}{\rho}\right\}
$$

The system $(C)$ is hyperbolic with all fields linearly degenerate.

Proof. Since $\mathcal{E}(t)$ is independent of time (during the timestep used for its resolution), the system (C) identifies with the original relaxation system (20) where we replace $\Pi$ by $\widetilde{\Pi}=\mathcal{E}^{2}(t) \Pi$, $a$ by $\widetilde{a}=\mathcal{E}(t) \sqrt{\chi_{c}} a$ and $b$ by $\widetilde{b}=\mathcal{E}(t) \sqrt{\chi_{c}} b$. It is thus hyperbolic with linearly degenerate characteristic fields.

Notice that if $\sqrt{\chi_{c}} a / \rho \sim c$ then in the low-Mach regime $\mathcal{E}(t) \sim M$, all the eigenvalues of the system $(C)$ are $O(|u|)$, and the convective system contains only the slow scale dynamics. Since according to (50) one has $0 \leq \chi_{c} \leq 1$, the previous assumption means that $a$ is of the order of $1 / M$ as $M \rightarrow 0$.

Lemma 4.2. The characteristic speeds of system $(A)$ are given by

$$
\Sigma_{A}=\left\{0,0,0,0,0,-\left(1-\mathcal{E}^{2}(t)\right) \sqrt{\chi_{a}} \frac{a}{\rho},+\left(1-\mathcal{E}^{2}(t)\right) \sqrt{\chi_{a}} \frac{a}{\rho}\right\} .
$$

The system $(A)$ is hyperbolic with all fields linearly degenerate.

The proof of this lemma is easy and not detailed here. Note that if $a$ is of the order of $1 / M$ as $M \rightarrow 0$ (as above), then all the eigenvalues of the system $(A)$ are of the order of the sound speed, which corresponds to the fast scale dynamics.

\subsection{Stability conditions for the splitting algorithm, and choice of $a, b, \chi_{c}, \chi_{a}$}

In order to define admissible relaxation parameters $a, b, \chi_{c}, \chi_{a}$, we have to write down stability conditions. We follow here the methodology introduced in [6], and we consider the relaxation limits of systems $(C)$ and $(A)$ separately.

Lemma 4.3. (i) Up to errors of the order $O\left(\Delta t^{2}\right)$, the solution to system $(C)$ starting at equilibrium and projected back at equilibrium after a timestep $\Delta t$ verifies

$$
\left\{\begin{aligned}
\partial_{t} \rho+\partial_{x}(\rho u) & =\frac{\Delta t}{2} \mathcal{E}^{2}(t) \partial_{x}\left(\left(\frac{a}{b}-1\right) \partial_{x} p\right), \\
\partial_{t}(\rho u)+\partial_{x}\left(\rho u^{2}+\mathcal{E}^{2}(t) p\right) & =\frac{\Delta t}{2} \mathcal{E}^{2}(t) \partial_{x}\left(\left(\frac{a}{b}-1\right) u \partial_{x} p+\beta_{c} \partial_{x} u\right), \\
\partial_{t} E+\partial_{x}\left(E u+\mathcal{E}^{2}(t) p u\right) & =\frac{\Delta t}{2} \mathcal{E}^{2}(t) \partial_{x}\left(\left(\frac{a}{b}-1\right) \frac{E+\mathcal{E}^{2}(t) p}{\rho} \partial_{x} p+\beta_{c} u \partial_{x} u\right),
\end{aligned}\right.
$$


with

$$
\beta_{c}=\chi_{c} \frac{a b}{\rho}-\rho\left(\frac{\partial p}{\partial \rho}\right)_{s}+\left(1-\mathcal{E}^{2}(t)\right) \frac{p}{\rho}\left(\frac{\partial p}{\partial e}\right)_{\rho} .
$$

(ii) Up to errors of the order $O\left(\Delta t^{2}\right)$, the solution to system (A) starting at equilibrium and projected back at equilibrium after a timestep $\Delta t$ verifies

$$
\left\{\begin{aligned}
\partial_{t} \rho & =0 \\
\partial_{t}(\rho u)+\left(1-\mathcal{E}^{2}(t)\right) \partial_{x} p & =\frac{\Delta t}{2}\left(1-\mathcal{E}^{2}(t)\right)^{2} \partial_{x}\left(\beta_{a} \partial_{x} u\right) \\
\partial_{t} E+\left(1-\mathcal{E}^{2}(t)\right) \partial_{x}(p u) & =\frac{\Delta t}{2}\left(1-\mathcal{E}^{2}(t)\right)^{2} \partial_{x}\left(\left(\frac{a}{b}-1\right) \frac{p}{\rho} \partial_{x} p+\beta_{a} u \partial_{x} u\right)
\end{aligned}\right.
$$

with

$$
\beta_{a}=\chi_{a} \frac{a b}{\rho}-\frac{p}{\rho}\left(\frac{\partial p}{\partial e}\right)_{\rho} .
$$

The proof of this lemma is very close to that of Lemma 3.2 (with $\varepsilon$ replaced here by $\Delta t / 2$ in accordance with [13]) and is not provided.

Lemma 4.4. Consider a viscosity of the form

$$
\left\{\begin{aligned}
\partial_{t} \rho & =\partial_{x}\left(\alpha \partial_{x} p\right) \\
\partial_{t}(\rho u) & =\partial_{x}\left(\alpha u \partial_{x} p+\beta \partial_{x} u\right) \\
\partial_{t} E & =\partial_{x}\left(\alpha \frac{E}{\rho} \partial_{x} p+\gamma u \partial_{x} u+\delta \frac{p}{\rho} \partial_{x} p\right)
\end{aligned}\right.
$$

with $E=\rho u^{2} / 2+\rho e$ and $\rho, p$, e are related by a state relation, and $\alpha, \beta, \gamma, \delta$ are (possibly state dependent) parameters. Then this viscous form is stable if and only if

$$
\beta \geq 0 \quad \text { and } \quad \alpha\left(\frac{\partial p}{\partial \rho}\right)_{e}+\delta \frac{p}{\rho^{2}}\left(\frac{\partial p}{\partial e}\right)_{\rho} \geq 0 .
$$

Proof. Denoting $U=(\rho, \rho u, E)$, the system can be written

$$
\partial_{t} U=\partial_{x}\left(D \partial_{x} U\right)
$$

with the matrix $D$ given by in terms of its lines by

$$
D=\left(\begin{array}{c}
\alpha p^{\prime} \\
\alpha u p^{\prime}+\beta u^{\prime} \\
\alpha \frac{E}{\rho} p^{\prime}+\gamma u u^{\prime}+\delta \frac{p}{\rho} p^{\prime}
\end{array}\right),
$$

where $p^{\prime}$ and $u^{\prime}$ stand for the derivatives of $p$ and $u$ with respect to $U$. These are linear forms, identified with line vectors. One can check that the eigenvalues of $D$ are

$$
0, \quad \frac{\beta}{\rho}, \quad, \alpha\left(\frac{\partial p}{\partial \rho}\right)_{e}+\delta \frac{p}{\rho^{2}}\left(\frac{\partial p}{\partial e}\right)_{\rho} .
$$

Writing that these eigenvalues are nonnegative we obtain (56). 
Note that Lemma 4.4 is a variant of Lemma 3.3. In Lemma 3.3 we have a particular form of (55) since the system (25) is (55) with $\gamma=\beta$ and $\delta=\alpha$. However in Lemma 3.3 it is required that $D^{t} \eta^{\prime \prime}$ is symmetric nonnegative, while here in Lemma 4.4 only the nonnegativity of the eigenvalues of $D$ is required. Notice that when $\delta=\alpha$, (56) reduces to (30) because

$$
\left(\frac{\partial p}{\partial \rho}\right)_{e}+\frac{p}{\rho^{2}}\left(\frac{\partial p}{\partial e}\right)_{\rho}=\left(\frac{\partial p}{\partial \rho}\right)_{e}+\left(\frac{\partial p}{\partial e}\right)_{\rho}\left(\frac{\partial e}{\partial \rho}\right)_{s}=\left(\frac{\partial p}{\partial \rho}\right)_{s}=c^{2}>0
$$

We deduce the stability conditions for the steps $(C)$ and $(A)$.

Lemma 4.5. (i) The relaxation limit of system $(C)$ is stable if and only if

$$
a \geq b, \quad \chi_{c} \frac{a b}{\rho^{2}} \geq\left(\frac{\partial p}{\partial \rho}\right)_{s}-\left(1-\mathcal{E}^{2}(t)\right) \frac{p}{\rho^{2}}\left(\frac{\partial p}{\partial e}\right)_{\rho} \geq 0 .
$$

(ii) The relaxation limit of system (A) is stable if and only if

$$
a \geq b, \quad \chi_{a} \frac{a b}{\rho^{2}} \geq \frac{p}{\rho^{2}}\left(\frac{\partial p}{\partial e}\right)_{\rho} .
$$

Proof. For (i), according to Lemma 4.3(i) the numerical viscosity in the relaxation limit of system $(C)$ takes the form (55) with $\alpha=a / b-1, \beta=\beta_{c}, \gamma=\beta_{c}$, and $\delta=(a / b-1) \mathcal{E}^{2}(t)$. Writing the stability conditions (56) we obtain $\beta_{c} \geq 0, a / b \geq 1$, and $\left(\frac{\partial p}{\partial \rho}\right)_{e}+\mathcal{E}^{2}(t) \frac{p}{\rho^{2}}\left(\frac{\partial p}{\partial e}\right)_{\rho} \geq 0$. Noticing that $\left(\frac{\partial p}{\partial \rho}\right)_{e}+\mathcal{E}^{2}(t) \frac{p}{\rho^{2}}\left(\frac{\partial p}{\partial e}\right)_{\rho}$ coincides with the expression in (61) by identity (60), and using the value (52) of $\beta_{c}$, we obtain (i).

For (ii), according to Lemma 4.3(ii) the numerical viscosity in the relaxation limit of system (A) takes the form (55) with $\alpha=0, \beta=\beta_{a}, \gamma=\beta_{a}$, and $\delta=(a / b-1)$. Writing the stability conditions (56) we obtain $\beta_{a} \geq 0$ and $a / b \geq 1$. With the value (54) of $\beta_{a}$ it yields (62).

We remark that the stability conditions (61), (62) can be interpreted as a splitting of the stability condition (30), since adding (61) to (62) multiplied by $1-\mathcal{E}^{2}(t)$ and using (50), we obtain (30).

For reasons that will be clear in Subsection 4.3.2 we would like to take $a$ and $b$ such that $a b / \rho$ and $a /(b \rho)$ are constant in space, which means that $a$ is proportional to $\rho$ and $b$ is constant. We are thus led to the following change of unknows: we consider that

$$
a=\rho \lambda, \quad b=\phi \lambda, \quad \chi_{c}=\frac{\lambda_{c}^{2}}{\lambda^{2}}, \quad \chi_{a}=\frac{\lambda_{a}^{2}}{\lambda^{2}},
$$

where $\phi, \lambda, \lambda_{c}, \lambda_{a}>0$ are four new parameters. Then we have

$$
\frac{a b}{\rho}=\phi \lambda^{2}, \quad \frac{a}{b \rho}=\frac{1}{\phi} .
$$

The stability conditions (61), (62) become then

$$
\rho \geq \phi, \quad \phi \lambda_{c}^{2} \geq \rho\left(\frac{\partial p}{\partial \rho}\right)_{s}-\left(1-\mathcal{E}^{2}(t)\right) \frac{p}{\rho}\left(\frac{\partial p}{\partial e}\right)_{\rho} \geq 0, \quad \phi \lambda_{a}^{2} \geq \frac{p}{\rho}\left(\frac{\partial p}{\partial e}\right)_{\rho} .
$$


We need in particular $\rho$ to be lower bounded away from 0 . A natural way to satisfy (65) is to take $\phi$ a lower bound on $\rho$, and then define $\lambda_{c}, \lambda_{a}$ large enough so as to satisfy (65). The last parameter $\lambda$ can be chosen finally so as to satisfy (50), i.e.

$$
\lambda^{2}=\lambda_{c}^{2}+\left(1-\mathcal{E}^{2}(t)\right) \lambda_{a}^{2}
$$

For a stiffened gas law $p=(\gamma-1) \rho e-\gamma p_{\infty}$ where $\gamma>1, p_{\infty} \geq 0$ are constants, the stability conditions (65) become

$$
\rho \geq \phi, \quad \phi \lambda_{c}^{2} \geq \frac{1+\mathcal{E}^{2}(t)(\gamma-1)}{\gamma} \rho c^{2}+\left(1-\mathcal{E}^{2}(t)\right)(\gamma-1) p_{\infty}, \quad \phi \lambda_{a}^{2} \geq \frac{\gamma-1}{\gamma} \rho c^{2}-(\gamma-1) p_{\infty}
$$

with $c^{2}=\gamma\left(p+p_{\infty}\right) / \rho$.

During the evolution, the speed parameters are managed as follows. The speeds $a, b$ are redefined at the beginning of each timestep (where here a timestep means a succession of the substeps of $(C),(A)$ and $(R))$ and are chosen according to (63)-(65). Indeed it is possible to choose $\phi, \lambda_{c}, \lambda_{a}$ so as to satisfy (65) for all relevant values of $\rho$ and $c$ that are attained during this timestep. We then define $\lambda$ by (66), and $b, \chi_{c}, \chi_{a}$ by (63). We observe however that there is a conflict for the value of $a$ during the convective substep, because $a=\rho \lambda$ defined by (63) with $\rho$ solving (47) will not satisfy $\partial_{t} a+v \partial_{x} a=0$. We thus consider that during the convection step, $a$ is transported according to $\partial_{t} a+v \partial_{x} a=0$. Then at the end of the convection step, $a$ is reinitialized as $a=\rho^{*} \lambda$ with $\rho^{*}$ the value of $\rho$ at the end of the acoustic step, before resolving the next (acoustic) substep. This reinitialization of $a$ does not alter the accuracy of the scheme, even for second-order versions.

With these definitions, when the Mach number $M$ is small, $a$ and $b$ are of the order of $1 / M$. It follows that $\delta_{1}$ in (25) is bounded, while $\delta_{2}$ is of the order of $1 / M^{2}$. But according to (8), (9), $p$ remains bounded whereas $\nabla \cdot \boldsymbol{u}$ is of the order of $M^{2}$, thus all the viscous terms in (25) remain bounded. Thus the viscosity in time due to the relaxation approximation does not grow when $M$ is small, which means that we do not loose accuracy in the low Mach number limit.

\subsection{Numerical schemes for each substep of the splitting}

In this subsection we present the space and time discretizations for the three substeps of the splitting method: convection, acoustic and relaxation steps.

\subsubsection{Numerical scheme for the convection step $(C)$}

For system $(C)$ i.e. (47), we consider an explicit finite volume scheme. For any quantity $g(t, x)$, we denote by $g_{j}^{n}$ an approximation of the average value of the quantity $g$ over the $j$-th cell at 
time $t_{n}$. The finite volume method reads

$$
\left\{\begin{aligned}
\rho_{j}^{*} & =\rho_{j}^{n}-\frac{\Delta t}{\Delta x}\left(F_{j+\frac{1}{2}}^{\rho}-F_{j-\frac{1}{2}}^{\rho}\right) \\
(\rho u)^{*} & =(\rho u)^{n}-\frac{\Delta t}{\Delta x}\left(F_{j+\frac{1}{2}}^{\rho u}-F_{j-\frac{1}{2}}^{\rho u}\right) \\
E^{*} & =E^{n}-\frac{\Delta t}{\Delta x}\left(F_{j+\frac{1}{2}}^{E}-F_{j-\frac{1}{2}}^{E}\right) \\
\Pi^{*} & =\Pi^{n}-\frac{\Delta t}{\Delta x}\left(F_{j+\frac{1}{2}}^{\pi}-F_{j-\frac{1}{2}}^{\pi}\right)-\frac{\Delta t}{\Delta x} F_{j}^{\Pi} \\
v^{*} & =v^{n}-\frac{\Delta t}{\Delta x}\left(F_{j+\frac{1}{2}}^{v}-F_{j-\frac{1}{2}}^{v}\right)-\frac{\Delta t}{\Delta x} F_{j}^{v}
\end{aligned}\right.
$$

with $\left(F_{j+\frac{1}{2}}^{\rho}\right), \ldots,\left(F_{j+\frac{1}{2}}^{v}\right)$ the conservative fluxes and $\left(F_{j}^{\Pi}\right),\left(F_{j}^{v}\right)$ the nonconservative fluxes. The quantities $\left(\rho^{*},(\rho u)^{*}, E^{*}, \Pi^{*}, v^{*}\right)$ are the values obtained at the end of this convective step.

We can consider the local Lax-Friedrichs (Rusanov) flux coupled with an upwind flux for the nonconservative part. It is also possible to design more accurate fluxes that are specific to the relaxation system. We consider a linear acoustic flux splitting similar to [25, 9]. Since according to Lemma 4.1 the system $(C)$ has all its fields linearly degenerate, we can also consider an explicit Godunov flux, as in [14]. These three fluxes are detailed in Appendix B and are compared numerically in Section 6.

\subsubsection{Numerical scheme for the acoustic step $(A)$}

For system $(A)$ that contains the fast scale dynamics, we consider an implicit scheme. In order to get higher accuracy we indeed propose a theta scheme. For any quantity $g$, we denote

$$
g^{n+\frac{1}{2}}=(1-\theta) g^{*}+\theta g^{* *},
$$

where $g^{*}$ is the value obtained at the end of the previous convective step and $g^{* *}$ is the value at the end of this acoustic step. The parameter $0 \leq \theta \leq 1$ is a constant. We consider the following semi-discretization in time,

$$
\left\{\begin{array}{l}
\rho^{* *}=\rho^{*}, \\
(\rho u)^{* *}=(\rho u)^{*}-\Delta t\left(1-\mathcal{E}^{2}(t)\right) \partial_{x} \Pi^{n+\frac{1}{2}}, \\
E^{* *}=E^{*}-\Delta t\left(1-\mathcal{E}^{2}(t)\right) \partial_{x}\left(\Pi^{n+\frac{1}{2}} v^{n+\frac{1}{2}}\right), \\
\Pi^{* *}+\theta \Delta t\left(1-\mathcal{E}^{2}(t)\right) \chi_{a} \frac{a^{*} b^{*}}{\rho^{*}} \partial_{x} v^{* *}=\Pi^{*}-(1-\theta) \Delta t\left(1-\mathcal{E}^{2}(t)\right) \chi_{a} \frac{a^{*} b^{*}}{\rho^{*}} \partial_{x} v^{*}, \\
v^{* *}+\theta \Delta t\left(1-\mathcal{E}^{2}(t)\right) \frac{a^{*}}{b^{*} \rho^{*}} \partial_{x} \Pi^{* *}=v^{*}-(1-\theta) \Delta t\left(1-\mathcal{E}^{2}(t)\right) \frac{a^{*}}{b^{*} \rho^{*}} \partial_{x} \Pi^{*} .
\end{array}\right.
$$

Then, plugging the equation on $v^{* *}$ into the equation on $\Pi^{* *}$, we get

$$
\Pi^{* *}-\theta^{2} \Delta t^{2}\left(1-\mathcal{E}^{2}(t)\right)^{2} \chi_{a} \frac{a^{*} b^{*}}{\rho^{*}} \partial_{x}\left(\frac{a^{*}}{b^{*} \rho^{*}} \partial_{x} \Pi^{* *}\right)=R\left(\Pi^{*}, v^{*}\right),
$$


where the right-hand side is given by

$$
R\left(\Pi^{*}, v^{*}\right)=\Pi^{*}+\theta(1-\theta) \Delta t^{2}\left(1-\mathcal{E}^{2}(t)\right)^{2} \chi_{a} \frac{a^{*} b^{*}}{\rho^{*}} \partial_{x}\left(\frac{a^{*}}{b^{*} \rho^{*}} \partial_{x} \Pi^{*}\right)-\Delta t\left(1-\mathcal{E}^{2}(t)\right) \chi_{a} \frac{a^{*} b^{*}}{\rho^{*}} \partial_{x} v^{*} .
$$

Here we observe that the quantities $a^{*} b^{*} / \rho^{*}=\phi \lambda^{2}, a^{*} /\left(b^{*} \rho^{*}\right)=1 / \phi$ are still constant in space. Consequently, and as desired, the elliptic equation (70) has constant coefficients.

Then we consider central fluxes to approximate both first and second-order derivatives at second-order accuracy. This is possible because the implicit time discretization ensures unconditional stability. For any discrete quantity $f=\left(f_{j}\right)_{j}$, we define

$$
D_{2 h}(f)=\left(\frac{f_{j+1}-f_{j-1}}{2 \Delta x}\right)_{j}, \quad D_{h^{2}}(f)=\left(\frac{f_{j+1}-2 f_{j}+f_{j-1}}{\Delta x^{2}}\right)_{j} .
$$

We first solve the $\Pi$ equation, which writes in the fully discrete case

$$
\left(\operatorname{Id}-\theta^{2} \Delta t^{2}\left(1-\mathcal{E}^{2}(t)\right)^{2} \chi_{a} \lambda^{2} D_{h^{2}}\right) \Pi^{* *}=R_{h}\left(\Pi^{*}, v^{*}\right),
$$

with

$$
R_{h}\left(\Pi^{*}, v^{*}\right)=\left(\operatorname{Id}+\theta(1-\theta) \Delta t^{2}\left(1-\mathcal{E}^{2}(t)\right)^{2} \chi_{a} \lambda^{2} D_{h^{2}}\right) \Pi^{*}-\Delta t\left(1-\mathcal{E}^{2}(t)\right) \chi_{a} \phi \lambda^{2} D_{2 h}\left(v^{*}\right) .
$$

Then we compute $\Pi^{n+\frac{1}{2}}=(1-\theta) \Pi^{*}+\theta \Pi^{* *}$ and we obtain $v^{* *}$ as

$$
v^{* *}=v^{*}-\Delta t\left(1-\mathcal{E}^{2}(t)\right) \frac{1}{\phi} D_{2 h}\left(\Pi^{n+\frac{1}{2}}\right),
$$

and $v^{n+\frac{1}{2}}=(1-\theta) v^{*}+\theta v^{* *}$. Finally $(\rho u)^{* *}$ and $E^{* *}$ are obtained by

$$
\begin{aligned}
(\rho u)^{* *} & =(\rho u)^{*}-\Delta t\left(1-\mathcal{E}^{2}(t)\right) D_{2 h}\left(\Pi^{n+\frac{1}{2}}\right), \\
E^{* *} & =E^{*}-\Delta t\left(1-\mathcal{E}^{2}(t)\right) D_{2 h}\left(\Pi^{n+\frac{1}{2}} v^{n+\frac{1}{2}}\right) .
\end{aligned}
$$

The acoustic step consists in solving successively (71), (72), (73), (74). Let us emphasize again that the implicit linear system to be solved (71) has constant coefficients.

\subsubsection{Numerical scheme for the relaxation step $(R)$}

The relaxation step is usually replaced by a projection step $[13,14,16]: \Pi^{n+1}=p^{* *}$ and $v^{n+1}=$ $u^{* *}$. In the following we rather consider the generalized projection step

$$
\left\{\begin{aligned}
\rho^{n+1} & =\rho^{* *}, \\
(\rho u)^{n+1} & =(\rho u)^{* *}, \\
E^{n+1} & =E^{* *}, \\
\Pi^{n+1} & =\Pi^{* *}+\omega\left(p^{* *}-\Pi^{* *}\right), \\
v^{n+1} & =v^{* *}+\omega\left(u^{* *}-v^{* *}\right),
\end{aligned}\right.
$$

with $\omega \in(0,2]$. When $\omega=1$ we recover the projection, and when $\omega>1$ we obtain the overrelaxation scheme studied in [17] and used in Lattice Boltzmann methods [7, 8]. It allows to reduce the numerical diffusion generated by the splitting method. 


\section{The scheme in several dimensions}

Here we extend our relaxation method to several dimensions. As presented in the introduction, the Euler system tends in the low-Mach limit to the incompressible Euler equations with advection of the density. Standard schemes generate a too large diffusion error on the density (as in the one-dimensional case) and also on the velocity field, which breaks the preservation of the divergence free constraint.

In several dimensions, generalizing (20), (21) we consider the relaxation system

$$
\left\{\begin{array}{l}
\partial_{t} \rho+\nabla \cdot(\rho \boldsymbol{v})=0 \\
\partial_{t}(\rho \boldsymbol{u})+\nabla \cdot(\rho \boldsymbol{u} \otimes \boldsymbol{v})+\nabla \Pi=0 \\
\partial_{t} E+\nabla \cdot(E \boldsymbol{v})+\nabla \cdot(\Pi v)=0 \\
\partial_{t}(\rho \Pi)+\nabla \cdot(\rho \Pi \boldsymbol{v})+a b \nabla \cdot \boldsymbol{v}=\frac{\rho}{\varepsilon}(p-\Pi), \\
\partial_{t}(\rho \boldsymbol{v})+\nabla \cdot(\rho \boldsymbol{v} \otimes \boldsymbol{v})+\frac{a}{b} \nabla \Pi=\frac{\rho}{\varepsilon}(\boldsymbol{u}-\boldsymbol{v}), \\
\partial_{t} a+\boldsymbol{v} \cdot \nabla a=0 \\
\partial_{t} b+\boldsymbol{v} \cdot \nabla b=0
\end{array}\right.
$$

where $E=\rho|\boldsymbol{u}|^{2} / 2+\rho e$ and $p \equiv p(\rho, e)$ is the state law. It leads to the following diffusive approximation of the Euler system

$$
\left\{\begin{array}{l}
\partial_{t} \rho+\nabla \cdot(\rho \boldsymbol{u})=\varepsilon \nabla \cdot\left(\left(\frac{a}{b}-1\right) \nabla p\right), \\
\partial_{t}(\rho \boldsymbol{u})+\nabla \cdot(\rho \boldsymbol{u} \otimes \boldsymbol{u})+\nabla p=\varepsilon \nabla \cdot\left(\left(\frac{a}{b}-1\right) \boldsymbol{u} \otimes \nabla p+\frac{a b-\rho^{2} c^{2}}{\rho} \nabla \cdot \boldsymbol{u} \mathrm{Id}\right) \\
\partial_{t} E+\nabla \cdot(E \boldsymbol{u}+p \boldsymbol{u})=\varepsilon \nabla \cdot\left(\left(\frac{a}{b}-1\right) \frac{E+p}{\rho} \nabla p+\frac{a b-\rho^{2} c^{2}}{\rho} \boldsymbol{u} \nabla \cdot \boldsymbol{u}\right) .
\end{array}\right.
$$

We observe that the diffusion operator in (77) involves fluxes proportional to the gradient of the pressure and the divergence of the velocity. Consequently, as in the $1 \mathrm{~d}$ case, since $a / b-1$ is bounded, $a b-\rho^{2} c^{2}$ is of the order of $1 / M^{2}$ and according to (8), (9), $p$ remains bounded whereas $\nabla \cdot \boldsymbol{u}$ is of the order of $M^{2}$, one has that all the viscous terms in (77) remain bounded. Thus the relaxation approximation does not introduce excessive diffusion in the low-Mach regime.

The numerical scheme to solve (76) is the direct extension of the one-dimensional one. It is based to the same splitting method. The first splitting step is the convection step, which consists in solving

$$
\left\{\begin{array}{l}
\partial_{t} \rho+\nabla \cdot(\rho \boldsymbol{v})=0 \\
\partial_{t}(\rho \boldsymbol{u})+\nabla \cdot(\rho \boldsymbol{u} \otimes \boldsymbol{v})+\mathcal{E}^{2}(t) \nabla \Pi=0 \\
\partial_{t} E+\nabla \cdot(E \boldsymbol{v})+\mathcal{E}^{2}(t) \nabla \cdot(\Pi \boldsymbol{v})=0, \\
\partial_{t} \Pi+\boldsymbol{v} \cdot \nabla \Pi+\phi \lambda_{c}^{2} \nabla \cdot \boldsymbol{v}=0 \\
\partial_{t} \boldsymbol{v}+\boldsymbol{v} \cdot \nabla \boldsymbol{v}+\frac{\mathcal{E}^{2}(t)}{\phi} \nabla \Pi=0
\end{array}\right.
$$


where $a$ and $b$ are still chosen satisfying (63)-(66). This system is solved using the one dimensional finite volume fluxes presented in Subsection 4.3.1 to compute the flux in the normal direction at each interface between two discretization cells. From the data $\left(\rho_{j}^{n},(\rho \boldsymbol{u})_{j}^{n}, E_{j}^{n}, \Pi_{j}^{n}, \boldsymbol{v}_{j}^{n}\right)$ at time $t^{n}$ and for all cell $j$, we denote $\left(\rho_{j}^{*},(\rho \boldsymbol{u})_{j}^{*}, E_{j}^{*}, \Pi_{j}^{*}, \boldsymbol{v}_{j}^{*}\right)$ the values obtained at the end of this first substep.

Then we solve the acoustic system

$$
\left\{\begin{array}{l}
\partial_{t} \rho=0, \\
\partial_{t}(\rho \boldsymbol{u})+\left(1-\mathcal{E}^{2}(t)\right) \nabla \Pi=0, \\
\partial_{t} E+\left(1-\mathcal{E}^{2}(t)\right) \nabla \cdot(\Pi \boldsymbol{v})=0, \\
\partial_{t} \Pi+\left(1-\mathcal{E}^{2}(t)\right) \phi \lambda_{a}^{2} \nabla \cdot \boldsymbol{v}=0, \\
\partial_{t} \boldsymbol{v}+\frac{\left(1-\mathcal{E}^{2}(t)\right)}{\phi} \nabla \Pi=0 .
\end{array}\right.
$$

As in the 1D case, we consider an implicit $\theta$-scheme for the elliptic equation satisfied by $\Pi$ obtained after plugging the $v$ equation into the $\Pi$ equation. Here, we also restrict ourselves to uniform Cartesian meshes in both spatial directions and consider second-order centered discretizations of the divergence and the gradient. Performing the same manipulations as in Subsection 4.3.2, we obtain the same numerical scheme (71), (72), (73), (74) to get $\left(\rho_{j}^{* *},(\rho \boldsymbol{u})_{j}^{* *}, E_{j}^{* *}, \Pi_{j}^{* *}, \boldsymbol{v}_{j}^{* *}\right)$ from $\left(\rho_{j}^{*},(\rho \boldsymbol{u})_{j}^{*}, E_{j}^{*}, \Pi_{j}^{*}, \boldsymbol{v}_{j}^{*}\right)$. We first solve the following discrete elliptic equation with constant coefficients

$$
\Pi_{j}^{* *}-\theta^{2} \Delta t^{2}\left(1-\mathcal{E}^{2}(t)\right)^{2} \lambda_{a}^{2} \operatorname{Div}_{h}\left(\nabla_{h} \Pi_{j}^{* *}\right)=R\left(\Pi_{j}^{*}, v_{j}^{*}\right)
$$

with

$$
R\left(\Pi_{j}^{*}, v_{j}^{*}\right)=\Pi_{j}^{*}+\theta(1-\theta) \Delta t^{2}\left(1-\mathcal{E}^{2}(t)\right)^{2} \lambda_{a}^{2} \operatorname{Div}_{h}\left(\nabla_{h} \Pi_{j}^{*}\right)-\Delta t\left(1-\mathcal{E}^{2}(t)\right) \phi \lambda_{a}^{2} \operatorname{Div}_{h} v_{j}^{*},
$$

where $\operatorname{Div}_{h}$ and $\nabla_{h}$ are the standard finite difference second-order discretizations of the divergence and the gradient operators. Then we compute $\Pi^{n+\frac{1}{2}}=(1-\theta) \Pi^{*}+\theta \Pi^{* *}$ and we obtain $v^{* *}$ with

$$
v_{j}^{* *}=v_{j}^{*}-\Delta t\left(1-\mathcal{E}^{2}(t)\right) \frac{1}{\phi} \nabla_{h} \Pi_{j}^{n+\frac{1}{2}},
$$

and compute $\boldsymbol{v}^{n+\frac{1}{2}}=(1-\theta) \boldsymbol{v}^{*}+\theta \boldsymbol{v}^{* *}$. Finally $(\rho \boldsymbol{u})^{* *}$ and $E^{* *}$ are obtained by

$$
\begin{aligned}
(\rho \boldsymbol{u})_{j}^{* *} & =(\rho \boldsymbol{u})_{j}^{*}-\Delta t\left(1-\mathcal{E}^{2}(t)\right) \nabla_{h} \Pi_{j}^{n+\frac{1}{2}}, \\
E_{j}^{* *} & =E_{j}^{*}-\Delta t\left(1-\mathcal{E}^{2}(t)\right) \operatorname{Div}_{h}\left(\Pi_{j}^{n+\frac{1}{2}} \boldsymbol{v}_{j}^{n+\frac{1}{2}}\right) .
\end{aligned}
$$

After that, the relaxation step is identical to the one-dimensional one (75). 


\section{Numerical results}

In this section we carry out several test cases to evaluate the numerical properties of our new semi-implicit scheme. It will be referred as the SI two-speed method (semi-implicit two-speed method).

We start with one-dimensional test-cases in Subsections 6.1, 6.2, 6.3 that show that the scheme well captures the material wave in the low-Mach regime. We compare the results with the schemes presented in the introductory section 2: implicit Jin-Xin method and SI Suliciu method (semi-implicit Suliciu method). We also compare these schemes with an explicit method.

Then we consider two-dimensional test-cases in Subsections 6.4 and 6.5. In two dimensions, the low-Mach regime is more difficult to capture as the velocity field is no longer necessarily constant but is approximately divergence free.

\subsection{Contact waves (1d)}

Steady contact wave. We first consider a steady contact discontinuity given by

$$
\rho(t, x)=\mathbb{I}_{x<x_{0}}+0.1 \mathbb{1}_{x>x_{0}}, \quad u(t, x)=0, \quad p(t, x)=1,
$$

where $x_{0}$ belongs to the computational domain and $\mathbb{I}$ denotes the characteristic function of a set. It is a steady solution to the Euler system. We consider the perfect gas law: $p=(\gamma-1) \rho e$ with $\gamma=1.4$. We solve the system on the time interval $\left[0, T_{f}\right]$ with $T_{f}=10$.

For all the numerical schemes except the implicit Jin-Xin scheme presented in Subsection 2.1 , the timestep is computed by the CFL condition associated with the explicit convection part of the scheme: $\Delta t=0.5 \frac{h}{\sup _{x} \lambda_{\max }(x)}$, where $h$ is the space step and $\lambda_{\max }(x)$ the maximal characteristic speed of the system. For the implicit Jin-Xin scheme we take $\Delta t=20 \frac{h}{\lambda_{\max }}$. The relaxation parameters are chosen as follows:

$$
\begin{aligned}
\text { Implicit Jin-Xin: } & \lambda=3.8, \\
\text { Semi-implicit Suliciu: } & \lambda_{c}=1.2, \lambda_{a}=0.8, \\
\text { Semi-implicit two-speed: } & \lambda_{c}=3.5, \lambda_{a}=2.6, \phi=0.099,
\end{aligned}
$$

and the splitting parameter $\mathcal{E}^{2}(t)$ is computed with (17), with $M_{\min }=10^{-4}$. We choose the classical first-order scheme $\omega=1$ and $\theta=1$.

We compare the accuracy of the different schemes in Table 1. We observe that the explicit Rusanov and the implicit Jin-Xin scheme are not accurate compared with the other schemes. Both the semi-implicit Suliciu (SI Suliciu) and the semi-implicit two-speed relaxation (SI twospeed) with the Rusanov solver for the explicit convection step provide better results. Using the linear acoustic flux splitting (FVS) or the Godunov scheme, we capture exactly the contact discontinuity.

In Table 2 we provide the timestep associated with the different schemes. We notice that since the velocity vanishes, $\mathcal{E}(t)$ takes the minimal value. This shows that the SI schemes allow to use larger timesteps than the implicit Jin-Xin method with a similar or higher accuracy. We observe 
also that the SI two speed relaxation scheme allows to use on this test case a timestep three times larger than the SI Suliciu method. This difference comes from to the eigenvalues. The factor $\frac{1}{\rho}$ in the maximal wave speed of the Suliciu relaxation model induces difficulties for strongly varying density with low density. We also remark that as expected the Xin-Jin implicit scheme is very diffusive since the time scheme admits an error homogeneous to $c$ on the density.

In Table 3 we compare the condition numbers of the matrices associated with the elliptic equations solved in the acoustic steps of the SI Suliciu and the SI two-speed relaxation schemes. For the test-case considered so far, the condition number is actually slightly larger for the SI two-speed (4.25e7) than for the SI Suliciu (3.75e7) but this is because the timestep is 3.5 larger. We obtain the same condition number for the two methods with a timestep 3 times larger for the SI two-speed relaxation method. For the same timestep, the condition number of the SI two-speed method is ten times smaller. Furthermore, since the matrix has constant coefficients in the SI two-speed relaxation method, it enables us to avoid assembling the matrix at each time iteration. This result shows that for this test case, the SI two-speed relaxation method is more efficient than the SI Suliciu one.

\begin{tabular}{|c|c|c|c|c|c|}
\cline { 3 - 6 } \multicolumn{2}{c|}{} & $\mathrm{N}=250$ & $\mathrm{~N}=500$ & $\mathrm{~N}=1000$ & $\mathrm{~N}=2000$ \\
\hline \multirow{2}{*}{ Explicit (Rusanov) } & error & 0.31 & 0.26 & 0.22 & 0.18 \\
& order & - & 0.25 & 0.24 & 0.29 \\
\hline \multirow{2}{*}{ Explicit (FVS) } & error & 0.0 & 0.0 & 0.0 & 0.0 \\
& order & - & - & - & - \\
\hline \multirow{2}{*}{ Implicit Jin-Xin } & error & 0.62 & 0.63 & 0.61 & 0.55 \\
& order & 0 & 0 & 0 & 0.15 \\
\hline \multirow{2}{*}{ SI Suliciu (Rusanov) } & error & $3.8 E^{-2}$ & $3.2 E^{-2}$ & $2.6 E^{-2}$ & $2.2 E^{-2}$ \\
& order & - & 0.25 & 0.3 & 0.24 \\
\hline \multirow{2}{*}{ SI two-speed (Rusanov) } & error & $3.4 E^{-2}$ & $3.0 E^{-2}$ & $2.6 E^{-2}$ & $2.2 E^{-2}$ \\
& order & - & 0.25 & 0.23 & 0.24 \\
\hline \multirow{2}{*}{ SI two-speed (FVS) } & error & 0.0 & 0.0 & 0.0 & 0.0 \\
& order & - & - & - & - \\
\hline \multirow{2}{*}{ SI two-speed (Godunov) } & error & 0.0 & 0.0 & 0.0 & 0.0 \\
& order & - & - & - & - \\
\hline
\end{tabular}

Table 1: (Stationary contact wave) Numerical error $\left(L^{2}\right.$ norm) as function of the number of cells $N$ for the different schemes.

Smooth contact wave. The second test is a moving smooth contact, with initial condition

$$
\rho(0, x)=0.1+\frac{1}{5 \sigma \sqrt{\pi}} e^{-\left(x-x_{0}\right)^{2} / \sigma^{2}}, \quad u(0, x)=0.002, \quad p(0, x)=1,
$$

with $\sigma=0.02$. We consider two different pressure laws: the perfect gas law and the stiffened gas law $p=(\gamma-1) \rho e-\gamma p_{\infty}$ with $p_{\infty}=10$ and $\gamma=1.4$. In both cases, the exact solution is the 


\begin{tabular}{|c|c|c|}
\cline { 2 - 3 } \multicolumn{1}{c|}{} & $\lambda_{\max }$ & $\Delta t$ \\
\hline Explicit & $\max (|u-c|,|u+c|)$ & $2.7 E^{-4}$ \\
\hline Implicit Jin-Xin & - & 0.0052 \\
\hline SI Suliciu & $\max \left(\left|u-\mathcal{E}(t) \lambda_{c} / \rho\right|,\left|u+\mathcal{E}(t) \lambda_{c} / \rho\right|\right)$ & 0.83 \\
\hline SI two-speed & $\max \left(\left|v-\mathcal{E}(t) \lambda_{c}\right|,\left|v+\mathcal{E}(t) \lambda_{c}\right|\right)$ & 2.8 \\
\hline
\end{tabular}

Table 2: (Stationary contact wave) Maximal characteristic speed $\lambda_{\max }$ of the explicit part and timesteps $\Delta t$ used in the stationary contact wave test-case for the different schemes.

\begin{tabular}{|c|c|c|}
\cline { 2 - 3 } \multicolumn{1}{c|}{} & $\Delta t$ & condition number \\
\hline SI Suliciu & 0.83 & $3.75 E^{7}$ \\
\hline SI two-speed & 2.8 & $4.25 E^{7}$ \\
\hline SI two-speed & 2.5 & $3.5 E^{7}$ \\
\hline SI two-speed & 0.82 & $3.7 E^{6}$ \\
\hline
\end{tabular}

Table 3: (Stationary contact wave) Condition numbers of the matrices of the discrete elliptic equations for the semi-implicit relaxation schemes.

advection of the density at constant velocity $u=0.002$. We solve the system up to the final time $T_{f}=2$.

The timestep is chosen as described in the previous test-case. The relaxation parameters are taken as follows:

$$
\begin{array}{rll} 
& \text { (perfect gas law) } & \text { (stiffened gas law) } \\
\text { Semi-implicit Suliciu: } & \lambda_{c}=3.2, \lambda_{a}=2.2, & \lambda_{c}=10, \lambda_{a}=6, \\
\text { Semi-implicit two-speed: } & \lambda_{c}=3.6, \lambda_{a}=3.2, \phi=0.098, & \lambda_{c}=12, \lambda_{a}=9, \phi=0.098 .
\end{array}
$$

As before we use $\omega=1$ and $\theta=1$.

Tables 4 and 5 show that the SI two-speed and the SI Suliciu method capture the transported density with the same accuracy as the explicit scheme with the FVS solver. Like Lagrangeremap solvers, the FVS solver has been actually constructed to accurately resolve the material wave. These results are obtained with the timestep showed in Table 6. For the SI two-speed method, the timestep is 1000 times larger than for the explicit scheme. We remark that it is also seven times larger that the timestep used by the SI Suliciu scheme. In Table 7 we show the condition numbers of the matrices associated with the elliptic equations in the implicit step of the semi-implicit schemes, when the number of cells is 1000 . We observe that using the same timestep, the condition number of the SI two-speed method is ten times smaller than for the SI Suliciu method. For the SI Suliciu method, the condition number is worsened by the large variation of the density (between 0.1 and 6) in this test case.

For the SI two-speed relaxation method, we now consider different values of the initial velocity $u_{0}$. We take the final time as $T_{f}=0.05 / u_{0}$. Consequently the density moves over the same distance for all initial velocities. The scheme can be considered as uniformly accurate in 
the low-Mach regime if the numerical error remains bounded as $u_{0}$ tends to 0 . Table 8 shows that the scheme is uniformly accurate and that the number of iterations is constant. The CPU cost is nearly constant as the Mach number decreases. Only the condition number of the implicit step and consequently the number of iterations of the linear solver may increase as the timestep increases. Indeed the elliptic operator is the sum of the identity and the Laplacian. When the Mach number tends to zero the operator tends to the Laplacian. Thus the condition number depends on the Mach number but is bounded by that of the Laplacian. However, using appropriate preconditioning like the multi-grid method, we could expect a uniform CPU cost whatever the Mach number is.

\begin{tabular}{|c|c|c|c|c|c|}
\cline { 3 - 6 } \multicolumn{2}{c|}{} & $N=250$ & $N=500$ & $N=1000$ & $N=2000$ \\
\hline \multirow{2}{*}{ Explicit (Rusanov) } & error & 0.77 & 0.67 & 0.53 & 0.38 \\
& order & - & 0.2 & 0.34 & 0.48 \\
\hline \multirow{2}{*}{ Explicit (FVS) } & error & $1.63 E^{-2}$ & $8.3 E^{-3}$ & $4.1 E^{-3}$ & $2.0 E^{-3}$ \\
& order & - & 0.96 & 1.02 & 1.03 \\
\hline \multirow{2}{*}{ SI Suliciu (Rusanov) } & error & $5.0 E^{-2}$ & $2.54 E^{-2}$ & $1.3 E^{-2}$ & $6.55 E^{-3}$ \\
& order & - & 0.97 & 0.98 & 0.99 \\
\hline \multirow{2}{*}{ SI two-speed (Rusanov) } & error & $1.1 E^{-1}$ & $6.5 E^{-2}$ & $3.4 E^{-2}$ & $1.7 E^{-2}$ \\
& order & - & 0.76 & 0.93 & 1.0 \\
\hline \multirow{2}{*}{ SI two-speed (FVS) } & error & $1.55 E^{-2}$ & $7.8 E^{-3}$ & $3.9 E^{-3}$ & $2.0 E^{-3}$ \\
& order & - & 0.99 & 1.0 & 1.0 \\
\hline \multirow{2}{*}{ SI two-speed (Godunov) } & error & $1.54 E^{-2}$ & $7.8 E^{-3}$ & $3.9 E^{-3}$ & $2.0 E^{-3}$ \\
& order & - & 1.0 & 1.0 & 1.0 \\
\hline
\end{tabular}

Table 4: (Smooth contact wave, Perfect Gas law) Numerical error ( $L^{2}$ norm) as function of the number of cells $N$ for the different schemes with the perfect gas law.

\subsection{Acoustic waves (1d)}

In this section we evaluate how the SI two-speed scheme captures acoustic waves.

Weakly colliding pulses. We consider the collision between two acoustic pulses in the low Mach regime. The initial condition is taken as

$$
\left\{\begin{array}{l}
\rho(t=0, x)=0.955+M_{\mathrm{ref}}\left(1-\cos \left(\frac{2 \pi x}{L}\right)\right) \\
u(t=0, x)=-\operatorname{sign}(x) \sqrt{\gamma}\left(1-\cos \left(\frac{2 \pi x}{L}\right)\right) \\
p(t=0, x)=\frac{1}{M_{\mathrm{ref}}^{2}}\left(1+\gamma M_{\mathrm{ref}}\left(1-\cos \left(\frac{2 \pi x}{L}\right)\right)\right),
\end{array}\right.
$$

over the domain $[-L, L]$ with $L=\frac{2}{M_{\text {ref }}}, M_{\text {ref }}=\frac{1}{11}$ and a perfect gas law with $\gamma=1.4$. Here it is interesting to recall a particular property of the gas dynamics equations. Denoting $\widetilde{p}=p-\bar{p}$ and 


\begin{tabular}{|c|c|c|c|c|c|}
\cline { 3 - 6 } \multicolumn{2}{l|}{} & $N=250$ & $N=500$ & $N=1000$ & $N=2000$ \\
\hline \multirow{2}{*}{ Explicit (Rusanov) } & error & 0.88 & 0.83 & 0.75 & 0.64 \\
& order & - & 0.1 & 0.15 & 0.23 \\
\hline \multirow{2}{*}{ Explicit (FVS) } & error & $1.63 E^{-2}$ & $8.3 E^{-3}$ & $4.1 E^{-3}$ & $2.0 E^{-3}$ \\
& order & - & 0.97 & 1.02 & 1.03 \\
\hline \multirow{2}{*}{ SI Suliciu (Rusanov) } & error & $4.85 E^{-2}$ & $2.4 E^{-2}$ & $1.2 E^{-2}$ & $6.25 E^{-3}$ \\
& order & - & 1.01 & 1.0 & 0.95 \\
\hline \multirow{2}{*}{ SI two-speed (Rusanov) } & error & 0.1 & $5.8 E^{-2}$ & $3.0 E^{-2}$ & $1.56 E^{-2}$ \\
& order & - & 0.78 & 0.95 & 0.95 \\
\hline \multirow{2}{*}{ SI two-speed (FVS) } & error & $1.5 E^{-2}$ & $7.8 E^{-3}$ & $3.9 E^{-3}$ & $1.95 E^{-3}$ \\
& order & - & 0.94 & 1.0 & 1.0 \\
\hline \multirow{2}{*}{ SI two-speed (Godunov) } & error & $1.5 E^{-2}$ & $7.8 E^{-3}$ & $3.9 E^{-3}$ & $1.97 E^{-3}$ \\
& order & - & 1.0 & 1.0 & 1.02 \\
\hline
\end{tabular}

Table 5: (Smooth contact wave, Stiffened Gas law) Numerical error ( $L^{2}$ norm) as function of the number of cells $N$ for the different schemes with the stiffened gas law.

\begin{tabular}{|c|c|c|c|}
\hline Scheme & $\lambda_{\max }$ & $\Delta t$ (PG law) & $\Delta t$ (SG law) \\
\hline Explicit & $\max (|u-c|,|u+c|)$ & $1.3 E^{-4}$ & $2.7 E^{-5}$ \\
\hline SI Suliciu & $\max \left(\left|u-\mathcal{E}(t) \lambda_{c} / \rho\right|,\left|u+\mathcal{E}(t) \lambda_{c} / \rho\right|\right)$ & 0.0038 & 0.004 \\
\hline SI two-speed & $\max \left(\left|v-\mathcal{E}(t) \lambda_{c}\right|,\left|v+\mathcal{E}(t) \lambda_{c}\right|\right)$ & 0.029 & 0.03 \\
\hline
\end{tabular}

Table 6: (Smooth contact wave) Maximal characteristic speed $\lambda_{\max }$ of the explicit part and timesteps $\Delta t$ used in the smooth contact wave test-case for the different schemes with the perfect gas law (PG law) and the stiffened gas law (SG law).

$\widetilde{e}=e+\bar{p} / \rho$ with $\bar{p}$ a constant, the gas dynamics equations for the state law $p(\rho, e)$ is equivalent to the gas dynamics equations for the state law $\widetilde{p}(\rho, \widetilde{e})$. When the state law $p(\rho, e)$ is a perfect gas law, one has that $\widetilde{p}(\rho, \widetilde{e})$ is a stiffened gas law with $p_{\infty}=\bar{p}$. Here taking $\bar{p}=1 / M_{\text {ref }}^{2}$, this algebraic transformation removes the constant value $1 / M_{\text {ref }}^{2}$ in the pressure, and we see that this test case can be better interpreted in terms of a stiffened gas law. The initial data are not well-prepared, thus non-negligible acoustic waves are emitted. We take $\omega=1$ and $\theta=1$ as previously, and the relaxation parameters are taken as

$$
\begin{aligned}
\text { Semi-implicit Suliciu: } & \lambda_{c}=12.5, \lambda_{a}=8.5, \\
\text { Semi-implicit two-speed: } & \lambda_{c}=12.0, \lambda_{a}=8.0, \phi=0.99,
\end{aligned}
$$

In Figure 1 (left) we compare the SI two-speed and the SI Suliciu methods computed with 1100 cells with the explicit Rusanov solution and a reference. We note that the two schemes capture the solution correctly. However the two relaxation semi implicit schemes are more diffusive that the explicit one. This numerical diffusion comes from the pressure derivative term $\partial_{x} p$ that appears in the diffusion operator on the three unknowns in (25), and that is not bounded 


\begin{tabular}{|c|c|c|}
\hline Schemes & $\Delta t$ & condition number \\
\hline SI Suliciu & 0.0038 & 23000 \\
\hline SI two-speed & 0.029 & 23000 \\
\hline SI two-speed & 0.0038 & 380 \\
\hline
\end{tabular}

Table 7: (Smooth contact wave) Condition numbers of the matrices of the discrete elliptic equations for the semi-implicit relaxation schemes (with 1000 cells).

\begin{tabular}{|c|c|c|c|c|}
\cline { 2 - 5 } \multicolumn{1}{c|}{} & \multicolumn{2}{c|}{ SI two-speed (Rusanov) } & \multicolumn{2}{c|}{ SI two-speed (FVS)/(Godunov) } \\
\cline { 2 - 5 } \multicolumn{1}{c|}{} & Error & $\Delta t$ & Error & $\Delta t$ \\
\hline$u_{0}=0.1$ & 0.26 & $6.0 E^{-4}$ & 0.046 & $6.0 E^{-4}$ \\
\hline$u_{0}=0.01$ & 0.26 & 0.006 & 0.046 & 0.006 \\
\hline$u_{0}=0.001$ & 0.26 & 0.06 & 0.046 & 0.06 \\
\hline
\end{tabular}

Table 8: (Smooth contact wave) Numerical errors (1000 cells).

here since the data are not well-prepared. In Figure 1 (right) we observe that the semi-implicit schemes for both Suliciu and two-speed relaxation methods allow to use timesteps 3 and 10 times larger than their explicit versions. Thus the use of semi-implicit schemes allows to reduce the CPU time. For this test case the CPU time for the explicit scheme is $8 \mathrm{~s}$ compared to $2.4 \mathrm{~s}$ for both semi-implicit schemes.
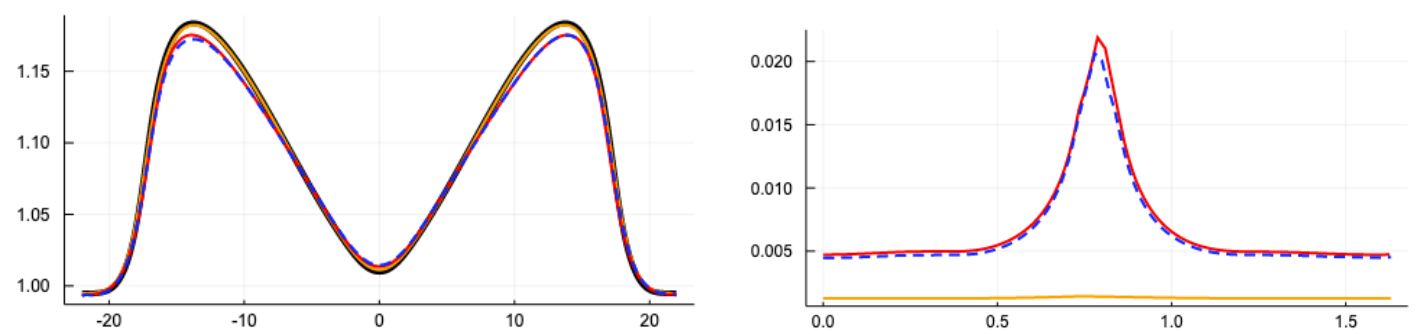

Figure 1: (Weakly colliding pulses.) Left: Density at time $t=1.63$ for the SI Suliciu (red), the SI two-speed method (blue) and for the explicit Rusanov (orange) with 2200 cells. Right: Evolution of the timestep $\Delta t$ over iterations for the explicit scheme (orange) and the semi-implicit Suliciu scheme (red) and semi-implicit two-speed scheme (blue).

Acoustic waves. We now consider a similar test case but with a larger Mach number. The initial data is given by

$\rho(t=0, x)=2.0+\frac{1}{20 \sqrt{\sigma}} e^{-\left(x-x_{0}\right)^{2} / \sigma^{2}}, \quad u(t=0, x)=0, \quad p(t=0, x)=1.0+\frac{1}{10 \sqrt{\sigma_{2}}} e^{-\left(x-x_{0}\right)^{2} / \sigma_{2}^{2}}$, 
on the domain [0,2] with $\sigma=0.02$ and $\sigma_{2}=0.08$. We take 800 cells, $\omega=1, \theta=1$ and the relaxation parameters

Semi-implicit Suliciu: $\quad \lambda_{c}=2, \lambda_{a}=1.4$,

Semi-implicit two-speed: $\quad \lambda_{c}=0.8, \lambda_{a}=0.55, \phi=1.98$.

At initial time the Mach number vanishes. It then increases and stabilizes around the value 0.6. If the parameter $M_{\min }$ in (17) is chosen too small, large timesteps are induced and the dynamics may not be well captured at the beginning. Thus we take $M_{\min }=0.1$. In Figure 2 we compare the SI Suliciu and SI two-speed relaxation schemes with the explicit Rusanov scheme. We observe on the density, velocity and pressure values that the two semi implicit schemes are a little more dissipative on acoustic waves. Thi is again because the schemes add dissipation in time on such waves. But the acoustic waves are captured with a good accuracy at the end. We also remark that the contact wave on density is captured with a very good accuracy compared to the classical explicit Rusanov scheme as expected. For this simulation the CPU time is $2.42 \mathrm{~s}$ for Rusanov, $1.85 \mathrm{~s}$ for Suliciu and $1.63 \mathrm{~s}$ for the two speed relaxation scheme. At the end for a CPU time divided by 1.5 the new scheme captures much better the contact wave and on the acoustic waves it has almost the same accuracy as the classical scheme. We remark also for this test case that the new relaxation method is a little bit more accurate than the Suliciu one.
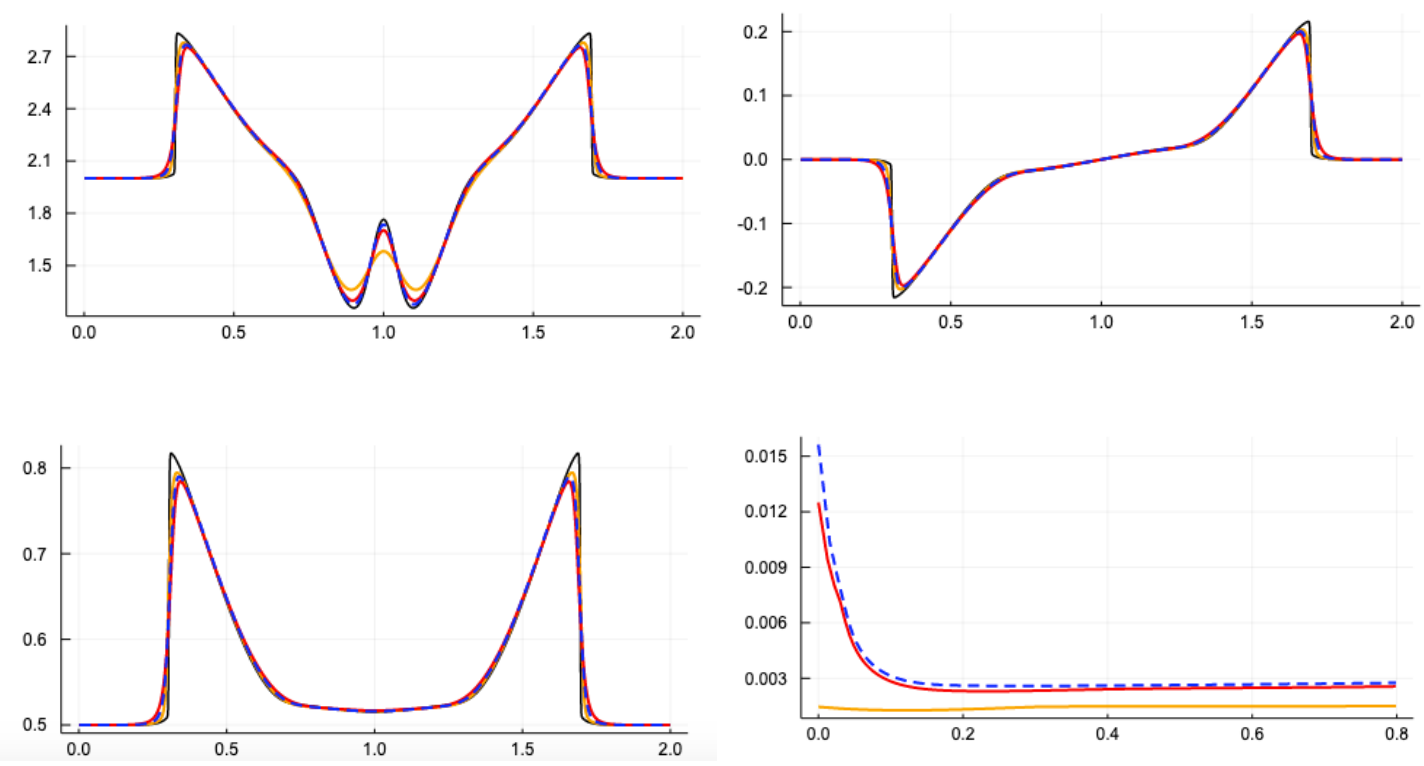

Figure 2: (Acoustic waves) Left Top: Density at time $t=0.8$ (800 cells). Right Top: velocity at time $t=0.8$ (800 cells). Left Bottom: Pressure at time $t=0.8$ (800 cells). Right Bottom. $\Delta t$ evolution. Black: fine solution, orange: Rusanov solution, red: SI Suliciu solution, blue: SI two-speed solution. 


\subsection{Shock tube test-case (1d)}

Mach variation. We consider a shock tube test-case with initial data

$$
\rho(t=0, x)=\mathbb{I}_{x<0}+0.125 \mathbb{I}_{x>0}, \quad u(t=0, x)=0, \quad p(t=0, x)=\mathbb{I}_{x<0}+(1-M) \mathbb{I}_{x>0},
$$

on the domain $\Omega=[-1,1]$ with the perfect gas law $(\gamma=1.4)$. As in the previous test case, one could also interpret the data as a stiffened gas law by removing the constant 1 to the pressure. The initial data are again not well prepared. We consider the following values of the Mach number: $M=0.9,0.2$, or 0.05 . We solve the Euler system up to the final time $T_{f}=0.3$ for $M=0.9$ and $T_{f}=0.15$ in the other cases (we reduce the final time because the waves go faster). For the semi-implicit Suliciu and two-speed relaxation schemes, we take $M_{\min }=M / 2$. We choose $\omega=1$ and $\theta=1$.

Tables $9(M=0.9), 10(M=0.2)$ and $11(M=0.05)$ show the numerical errors obtained for the different schemes on the three unknowns $\rho, u$ and $p$ when varying the number of cells $N$. The first remark is that, as in the previous test-cases, the classical Rusanov scheme is the less accurate scheme for the density while the relaxation schemes are the most accurate (especially the schemes based of FVS or Godunov solvers for the two-speed relaxation). Table 12 gives the timestep used for the different schemes.

Contrary to the contact wave test-cases (Subsection 6.1), the solution involves pressure and velocity discontinuities, which tend to be smoothed by the implicit part of the schemes. For $M=0.2$ in Table 10, we remark that the SI two-speed schemes (FSV and Godunov) and the explicit FVS scheme are 1.8 times more precise than the explicit scheme (Rusanov). They are approximately $45 \%$ less accurate on the velocity and $20 \%$ for the pressure but they use a timestep 6 times larger. For $M=0.05$ (Table 11), the SI two-speed schemes (FSV and Godunov) and the explicit FVS scheme are 3 times more precise on the density than the explicit scheme (Rusanov). They are approximately twice less accurate on the velocity and twice less for the pressure but this is using a timestep 25 times larger.

The SI two-speed scheme has the expected behavior. In the low-Mach regime, as the timestep is large compared to the time scale of the acoustic waves, these latter are not well resolved and large errors are made on the velocity and the pressure. Nevertheless we still keep a good accuracy on the density. These results show that the scheme allows to filter the fast scale dynamics while keeping a good accuracy on the slow scale one.

For a Mach number close to $1(M=0.9)$ in Table 9, the SI two-speed method (Godunov/FVS) has almost the same accuracy as the explicit (Rusanov) scheme. We also note that the SI Suliciu (Rusanov) and SI two speed (Rusanov) have the same accuracy on the density. The SI Suliciu (Rusanov) is yet more accurate on the pressure and less accurate on the velocity. However, the solution with the SI Suliciu scheme uses a timestep 3 times smaller.

Table 13 shows the condition numbers of the matrices involved in the implicit part of the schemes. As for the contact wave test-case, the condition number is smaller for the SI twospeed method than for the SI Suliciu one at equal timestep. This implies a reduction of the number of iterations of the iterative solvers of the associated linear system. Conversely, taking the same number of iterations, this enables to use larger timesteps.

Finally, concerning the spatial solver, the results indicate that in low-Mach regime $(M=$ 0.05), the SI two-speed with FVS is more accurate that the SI two-speed with Godunov or 
Rusanov solvers. However, it seems a little bit less stable. For a Mach number close to one, using the Godunov solver leads to a better accuracy.

\begin{tabular}{|c|c|c|c|c|c|c|c|}
\cline { 3 - 8 } \multicolumn{2}{c|}{} & \multicolumn{2}{c|}{ Explicit } & Suliciu & \multicolumn{3}{c|}{ Two speed relaxation } \\
\cline { 3 - 8 } \multicolumn{2}{c|}{} & Rusanov & FVS & Rusanov & Rusanov & Godunov & FVS \\
\hline \multirow{3}{*}{$N=250$} & $\rho$ & $3.66 E^{-2}$ & $3.34 E^{-2}$ & $4.55 E^{-2}$ & $4.81 E^{-2}$ & $3.37 E^{-2}$ & $3.53 E^{-2}$ \\
& $u$ & $1.02 E^{-1}$ & $1.00 E^{-1}$ & $1.92 E^{-1}$ & $1.58 E^{-1}$ & $1.16 E^{-1}$ & $1.18 E^{-1}$ \\
& $p$ & $3.70 E^{-2}$ & $3.6 E^{-2}$ & $4.93 E^{-2}$ & $5.37 E^{-2}$ & $3.7 E^{-2}$ & $3.93 E^{-2}$ \\
\hline \multirow{3}{*}{$N=500$} & $\rho$ & $2.73 E^{-2}$ & $2.40 E^{-2}$ & $3.54 E^{-2}$ & $3.65 E^{-2}$ & $2.49 E^{-2}$ & $2.62 E^{-2}$ \\
& $u$ & $6.70 E^{-2}$ & $6.65 E^{-2}$ & $1.50 E^{-2}$ & $1.21 E^{-1}$ & $9.36 E^{-2}$ & $9.53 E^{-2}$ \\
& $p$ & $2.48 E^{-2}$ & $2.37 E^{-2}$ & $3.52 E^{-2}$ & $3.81 E^{-2}$ & $2.58 E^{-2}$ & $2.75 E^{-2}$ \\
\hline \multirow{3}{*}{$N=1000$} & $\rho$ & $2.01 E^{-2}$ & $1.75 E^{-2}$ & $2.67 E^{-2}$ & $2.70 E^{-2}$ & $1.79 E^{-2}$ & $1.88 E^{-2}$ \\
& $u$ & $5.09 E^{-2}$ & $4.91 E^{-2}$ & $1.05 E^{-1}$ & $8.00 E^{-2}$ & $5.78 E^{-2}$ & $5.88 E^{-2}$ \\
& $p$ & $1.65 E^{-2}$ & $1.5 E^{-2}$ & $2.24 E^{-2}$ & $2.59 E^{-2}$ & $1.68 E^{-2}$ & $1.80 E^{-2}$ \\
\hline
\end{tabular}

Table 9: (Shock tube, $M=0.9)$ Numerical errors $\left(L^{2}\right.$ norm) at final time $T_{f}=0.3$. For the SI two-speed method: $\lambda_{c}=3.2, \lambda_{a}=2.6$ and $\phi=0.099$. For the SI Suliciu method: $\lambda_{c}=1.5$, $\lambda_{a}=1.2$.

\begin{tabular}{|c|c|c|c|c|c|c|c|}
\cline { 3 - 8 } \multicolumn{2}{c|}{} & \multicolumn{2}{c|}{ Explicit } & SI Suliciu & \multicolumn{3}{c|}{ SI two speed } \\
\cline { 3 - 8 } \multicolumn{2}{c|}{} & Rusanov & FVS & Rusanov & Rusanov & Godunov & FVS \\
\hline \multirow{3}{*}{$N=250$} & $\rho$ & $8.29 E^{-2}$ & $4.64 E^{-2}$ & $6.31 E^{-2}$ & $6.15 E^{-2}$ & $5.32 E^{-2}$ & $5.17^{-2}$ \\
& $u$ & $1.61 E^{-1}$ & $1.55 E^{-1}$ & $2.68 E^{-1}$ & $2.51 E^{-1}$ & $2.44 E^{-1}$ & $2.15 E^{-1}$ \\
& $p$ & $1.03 E^{-2}$ & $9.83 E^{-3}$ & $1.19 E^{-2}$ & $1.56 E^{-2}$ & $1.48 E^{-2}$ & $1.37 E^{-2}$ \\
\hline \multirow{3}{*}{$N=500$} & $\rho$ & $6.83 E^{-2}$ & $3.48 E^{-2}$ & $4.94 E^{-2}$ & $4.92 E^{-2}$ & $3.61 E^{-2}$ & $3.57 E^{-2}$ \\
& $u$ & $1.29 E^{-1}$ & $1.24 E^{-1}$ & $2.22 E^{-1}$ & $2.05 E^{-1}$ & $1.99 E^{-1}$ & $1.74 E^{-1}$ \\
& $p$ & $8.13 E^{-3}$ & $7.83 E^{-3}$ & $9.46 E^{-3}$ & $1.24 E^{-2}$ & $1.21 E^{-2}$ & $1.12 E^{-2}$ \\
\hline \multirow{3}{*}{$N=1000$} & $\rho$ & $5.72 E^{-2}$ & $2.94 E^{-2}$ & $4.12 E^{-2}$ & $4.12 E^{-2}$ & $2.97 E^{-2}$ & $2.95 E^{-2}$ \\
& $u$ & $1.00 E^{-1}$ & $9.60 E^{-2}$ & $1.79 E^{-2}$ & $1.62 E^{-1}$ & $1.57 E^{-1}$ & $1.36 E^{-1}$ \\
& $p$ & $6.30 E^{-3}$ & $6.05 E^{-3}$ & $7.53 E^{-3}$ & $1.00 E^{-2}$ & $9.74 E^{-3}$ & $9.00 E^{-3}$ \\
\hline
\end{tabular}

Table 10: (Shock tube, $M=0.2)$ Numerical errors $\left(L^{2}\right.$ norm) at final time $T_{f}=0.15$. For the SI two-speed method: $\lambda_{c}=3.0, \lambda_{a}=2.5$ and $\phi=0.099$. For the SI Suliciu method: $\lambda_{c}=1.5$, $\lambda_{a}=1.2$.

High-order extension In the previous test-case, we observe that the SI two-speed scheme computes less accurately the acoustic dynamics in the low-Mach regime. This mainly results from the errors generated by the first-order in time schemes for the relaxation and acoustic parts as well as the use of a large timestep. The two processes induce diffusion errors in time on the acoustic waves. 


\begin{tabular}{|c|c|c|c|c|c|c|c|}
\cline { 3 - 8 } \multicolumn{2}{c|}{} & \multicolumn{2}{c|}{ Explicit } & SI Suliciu & \multicolumn{3}{c|}{ SI two-speed } \\
\cline { 3 - 8 } \multicolumn{2}{c|}{$N=250$} & Rusanov & FVS & Rusanov & Rusanov & Godunov & FVS \\
\hline \multirow{3}{*}{$N=500$} & & $9.02 E^{-2}$ & $4.25 E^{-2}$ & $5.25 E^{-2}$ & $5.24 E^{-2}$ & $5.54 E^{-2}$ & $5.25 E^{-2}$ \\
& $u$ & $1.49 E^{-1}$ & $1.43 E^{-1}$ & $3.06 E^{-1}$ & $3.09 E^{-1}$ & $3.19 E^{-1}$ & $3.21 E^{-1}$ \\
& $p$ & $2.71 E^{-3}$ & $2.63 E^{-3}$ & $4.59 E^{-3}$ & $5.56 E^{-3}$ & $6.70 E^{-3}$ & $6.10 E^{-3}$ \\
\hline \multirow{3}{*}{$N=1000$} & $\rho$ & $7.44 E^{-2}$ & $2.50 E^{-2}$ & $3.74 E^{-2}$ & $3.67 E^{-2}$ & $2.54 E^{-2}$ & $2.49 E^{-2}$ \\
& $u$ & $1.07 E^{-1}$ & $1.03 E^{-1}$ & $2.41 E^{-2}$ & $2.27 E^{-1}$ & $2.28 E^{-2}$ & $2.08 E^{-1}$ \\
& $p$ & $2.15 E^{-3}$ & $2.09 E^{-3}$ & $3.52 E^{-3}$ & $4.40 E^{-3}$ & $4.40 E^{-2}$ & $4.15 E^{-3}$ \\
\hline & $\rho$ & $6.23 E^{-2}$ & $2.21 E^{-2}$ & $3.11 E^{-2}$ & $3.10 E^{-2}$ & $2.11 E^{-2}$ & $2.21 E^{-2}$ \\
& $u$ & $7.35 E^{-2}$ & $7.12 E^{-2}$ & $1.71 E^{-2}$ & $1.58 E^{-1}$ & $1.59 E^{-1}$ & $1.41 E^{-1}$ \\
& $p$ & $1.75 E^{-3}$ & $1.7 E^{-3}$ & $2.84 E^{-3}$ & $3.68 E^{-3}$ & $3.67 E^{-3}$ & $3.42 E^{-3}$ \\
\hline
\end{tabular}

Table 11: (Shock tube, $M=0.05)$ Numerical errors $\left(L^{2}\right.$ norm) at final time $T_{f}=0.15$. For the SI two-speed method: $\lambda_{c}=3.2, \lambda_{a}=2.6$ and $\phi=0.099$. For the SI Suliciu method: $\lambda_{c}=1.5$, $\lambda_{a}=1.2$.

\begin{tabular}{|c|c|c|c|c|}
\cline { 2 - 5 } \multicolumn{1}{c|}{} & $\lambda_{\max }$ & $\Delta t(M=0.9)$ & $\Delta t(M=0.2)$ & $\Delta t(M=0.05)$ \\
\hline Explicit & $\max (|u-c|,|u+c|)$ & $9.12 E^{-4}$ & $6.3 E^{-4}$ & $6.0 E^{-4}$ \\
\hline SI Suliciu & $\max \left(\left|u-\mathcal{E}(t) \lambda_{c} / \rho\right|,\left|u+\mathcal{E}(t) \lambda_{c} / \rho\right|\right)$ & $2.2 E^{-4}$ & $1.4 E^{-3}$ & $6.0 E^{-3}$ \\
\hline SI two-speed & $\max \left(\left|v-\mathcal{E}(t) \lambda_{c}\right|,\left|v+\mathcal{E}(t) \lambda_{c}\right|\right)$ & $5.2 E^{-4}$ & $5.5 E^{-3}$ & $1.6 E^{-2}$ \\
\hline
\end{tabular}

Table 12: (Shock tube) Timesteps $\Delta t$ for the different Mach number $M$ and for the different schemes for $N=500$ cells.

\begin{tabular}{|c|c|c|c|c|}
\cline { 3 - 5 } \multicolumn{2}{c|}{} & $\Delta t$ & Condition number & Nb iterations for convergence \\
\hline \multirow{3}{*}{$\mathrm{M}=0.2$} & SI Suliciu & $1.4 E^{-3}$ & $45-50$ & $70-85$ \\
& SI two-speed & $5.5 E^{-3}$ & $40-55$ & $70-85$ \\
& SI two-speed & $1.5 E^{-3}$ & $3-3.6$ & $19-22$ \\
\hline \multirow{3}{*}{$\mathrm{M}=0.05$} & SI Suliciu & $6.0 E^{-3}$ & $570-700$ & $290-700$ \\
& SI two-speed & $1.6 E^{-2}$ & $250-350$ & $210-520$ \\
& SI two-speed & $6.0 E^{-3}$ & $35-52$ & $75-90$ \\
\hline
\end{tabular}

Table 13: (Shock tube) Condition numbers and number of iterations to reach convergence for the two semi-implicit relaxation schemes.

In order to better capture the acoustic dynamics, we implement the following higher-order extension of the SI two-speed method. The explicit transport part is solved with a SPP-RK2 scheme and a MUSCL strategy [1, 19]. For the implicit acoustic part, the parameter $\theta$ is taken as $0.5 \leqslant \theta<1$. For $\theta=0.5$ we get the second-order Crank-Nicolson scheme. Finally, for the relaxation part, the parameter $\omega$ can be taken strictly larger than 1 .

However, when approaching second-order accuracy with $\theta<1$ and $\omega>1$, the scheme 
generates oscillations, that are larger for larger timesteps. These oscillations may induce instabilities. Consequently, taking a smaller timestep is necessary if one wants to capture low Mach acoustic waves.

In Figure 3 we compare the second-order explicit scheme (with SPP-RK2/MUSCL), the SI two-speed scheme with only a second-order explicit transport part and the SI two-speed scheme with second-order explicit transport part and $\theta=0.7, \omega=2$. We consider the previous Shock Tube test-case with $M=0.05$. In order to get a smaller timestep we take $M_{\min }=0.2$. Consequently the solution obtained with the semi-implicit scheme uses a 5 times larger timestep than the explicit scheme instead of 20 times in the results of Table 11.

All three schemes correctly capture the contact wave. The explicit scheme is the most accurate on the acoustic waves. The full second-order SI two-speed scheme is almost as accurate as the explicit scheme but the result is obtained with 5 times less time iterations. As regards the CPU time, this scheme is three times faster than the explicit one while obtaining a similar accuracy. Indeed the implicit solver converges quite fast at each iteration and the main cost comes from the explicit MUSCL part.

Note that the scheme is not fully second-order accurate since $\theta=0.7$ instead of 0.5 . Considering smaller values of $\theta$ generates a lot of oscillations. However, considering the scheme with intermediate $\theta$ and taking a smaller timestep but still larger than what the CFL condition would demand, we get a numerical method that is able to capture the acoustic waves and which is less costly than the explicit one .

\subsection{Smooth contact wave (2d)}

The first two-dimensional test case concerns the resolution of contact waves in the low-mach regime.

With a constant velocity field. We first consider a moving smooth contact wave with constant velocity. The initial data are given by

$$
\rho(t=0, \mathbf{x})=0.1+\frac{1}{5 \sigma \sqrt{\pi}} e^{-\left\|\mathbf{x}-\mathbf{x}_{0}\right\|^{2} / 2 \sigma^{2}}, \quad u_{1}(t=0, \mathbf{x})=u_{2}(t=0, \mathbf{x})=0.01, \quad p(t=0, \mathbf{x})=1,
$$

on the domain $\Omega=[0,1]^{2}$ with $\sigma=0.05$ and the perfect gas law $(\gamma=1.4)$. The solution to this problem consists in the advection of the density at constant velocity $\mathbf{u}_{0}$. The numerical solution is computed up to time $T_{f}=1$. As for the $1 \mathrm{D}$ case we use $\theta=1$ and $\omega=1$. For the relaxation parameters we take

$$
\begin{aligned}
\text { Semi-implicit Suliciu: } & \lambda_{c}=1.8, \lambda_{a}=1.3, \\
\text { Semi-implicit two-speed: } & \lambda_{c}=3.2, \lambda_{a}=2.5, \phi=0.099 .
\end{aligned}
$$

In Table 14 we compare the numerical errors obtained with the explicit, the SI Suliciu and the SI two-speed schemes. The results are very similar to those obtained in the one-dimensional test-cases. The SI schemes are able to capture the slow density transport and the schemes with the flux splitting solver (FVS) are the most accurate. 

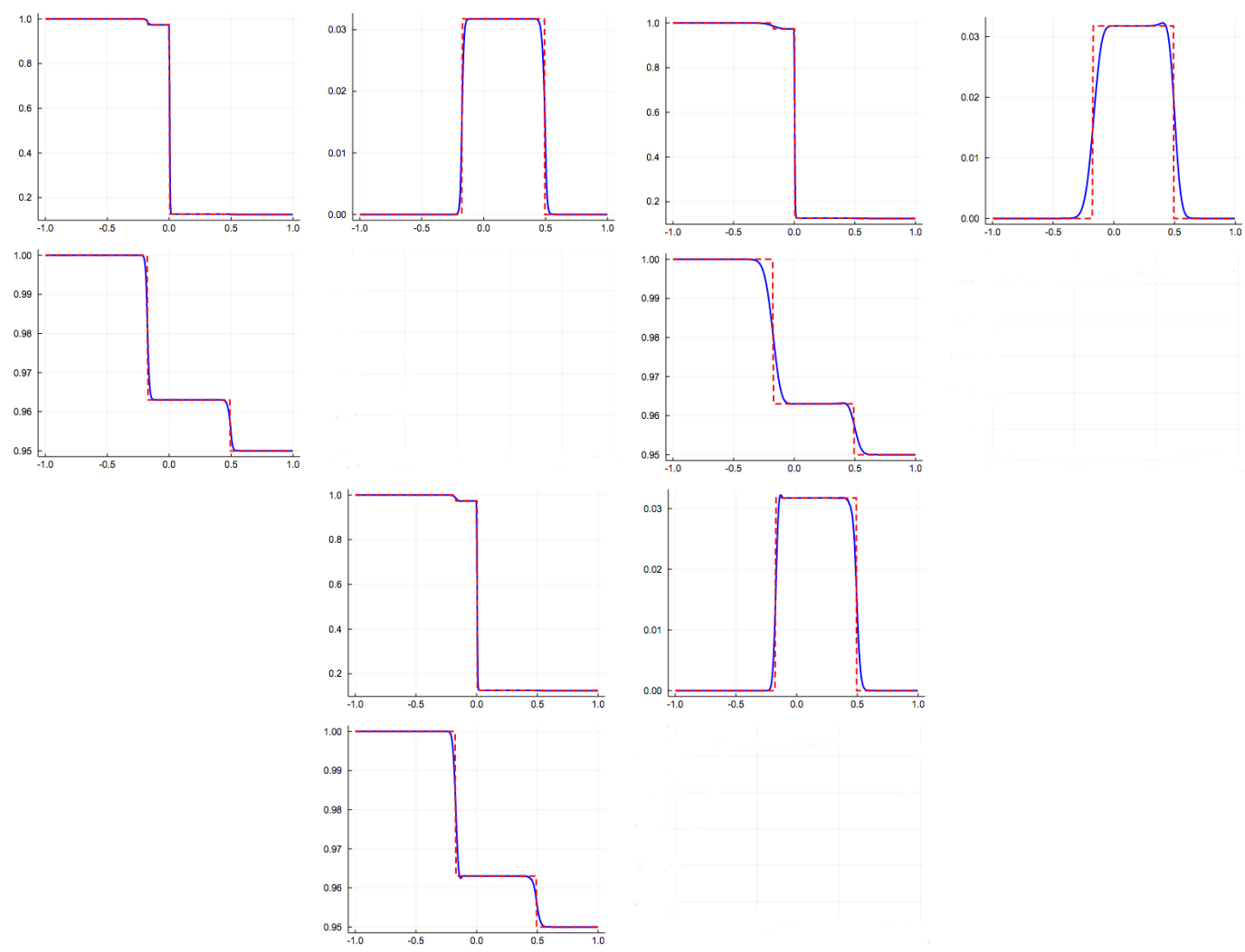

Figure 3: (Shock tube, $M=0.05$, high-order extension) Left: Second-order explicit scheme with FVS solver $(\rho, u$ and $p$ ). Right: SI two-speed scheme with second-order Godunov solver $(\rho, u$ and $p$ ). Parameters: $\theta=1, \omega=1$. Bottom: Semi-implicit second order Godunov scheme $(\rho, u$ and $p)$. Parameters: $\theta=0.7, \omega=2.0$. Relaxation parameters: $\lambda_{c}=3.2, \lambda_{a}=2.6$ and $\phi=0.099$. Computed with $N=500$ cells.

As in 1D test-cases we also observe in Table 15 that for a similar accuracy the SI two-speed scheme enables us to take a 40 times larger timestep than the one of the explicit scheme and 1.5 times larger than the one of the SI Suliciu.

Table 16 shows that we get the same kind of result as in the 1D case concerning the conditions numbers of the matrices of the implicit elliptic solver. By removing the spatial dependency on the density in the elliptic solver, the SI two-speed relaxation method enables a division by 3.3 of the condition number (at given timestep). 


\begin{tabular}{|c|c|c|c|c|c|c|c|c|c|c|}
\cline { 2 - 12 } \multicolumn{1}{c|}{} & \multicolumn{4}{c|}{ Explicit } & \multicolumn{2}{c|}{ SI Suliciu } & \multicolumn{4}{c|}{ SI two-speed } \\
\cline { 2 - 12 } \multicolumn{1}{c|}{ Rusanov } & \multicolumn{2}{c|}{ FVS } & \multicolumn{2}{c|}{ Rusanov } & \multicolumn{2}{c|}{ Rusanov } & \multicolumn{2}{c|}{ FVS } \\
\hline$N$ & Error & Order & Error & Order & Error & Order & Error & Order & Error & Order \\
\hline 100 & 0.078 & - & 0.045 & - & 0.19 & - & 0.22 & - & 0.042 & - \\
\hline 200 & 0.71 & $\mathrm{x}$ & 0.023 & $\mathrm{x}$ & 0.10 & $\mathrm{x}$ & 0.13 & $\mathrm{x}$ & 0.021 & $\mathrm{x}$ \\
\hline 400 & 0.58 & $\mathrm{x}$ & 0.011 & $\mathrm{x}$ & 0.051 & $\mathrm{x}$ & 0.071 & $\mathrm{x}$ & 0.011 & $\mathrm{x}$ \\
\hline 800 & 0.41 & $\mathrm{x}$ & 0.0058 & $\mathrm{x}$ & 0.026 & $\mathrm{x}$ & 0.038 & $\mathrm{x}$ & 0.0055 & $\mathrm{x}$ \\
\hline
\end{tabular}

Table 14: (Smooth contact wave, constant velocity, 2d) Numerical errors ( $L^{2}$ norm) for the different schemes.

\begin{tabular}{|c|c|c|}
\hline & $\lambda_{\max }$ & $\Delta t$ \\
\hline Explicit & $\max (|(\boldsymbol{u}, \mathbf{n})-c|,|(\boldsymbol{u}, \mathbf{n})+c|)$ & $2.1 E^{-3}$ \\
\hline SI Suliciu & $\max \left(\left|(\boldsymbol{u}, \mathbf{n})-\mathcal{E}(t) \lambda_{c} / \rho\right|,\left|(\boldsymbol{u}, \mathbf{n})+\mathcal{E}(t) \lambda_{c} / \rho\right|\right)$ & 0.056 \\
\hline SI two-speed & $\max \left(\left|(\boldsymbol{v}, \mathbf{n})-\mathcal{E}(t) \lambda_{c}\right|,\left|(\boldsymbol{v}, \mathbf{n})+\mathcal{E}(t) \lambda_{c}\right|\right)$ & 0.088 \\
\hline
\end{tabular}

Table 15: (Smooth contact wave, constant velocity, 2d) Maximal characteristic speed $\lambda_{\max }$ of the explicit part and timestep $\Delta t$ used for the different numerical schemes, for $N=200$ cells.

\begin{tabular}{|c|c|c|}
\cline { 2 - 3 } \multicolumn{1}{c|}{} & $\Delta t$ & conditioning \\
\hline SI Suliciu & 0.0575 & 2400 \\
\hline SI two-speed & 0.088 & 1750 \\
\hline SI two-speed & 0.057 & 720 \\
\hline
\end{tabular}

Table 16: (Smooth contact wave, constant velocity, 2d) Condition numbers for the two semiimplicit relaxation scheme for $N=200$ cells.

With a divergence free velocity field. We now consider a moving smooth contact with a divergence free velocity field. The initial data is given by

$$
\left\{\begin{array}{l}
\rho(t=0, \mathbf{x})=0.1+\frac{1}{5 \sigma \sqrt{\pi}} e^{-\left\|\mathbf{x}-\mathbf{x}_{0}\right\|^{2} / 2 \sigma^{2}} \\
u_{1}(t=0, \mathbf{x})=-0.001\left(y-y_{0}\right) \frac{1}{\sigma \sqrt{\pi}} e^{-\left\|\mathbf{x}-\mathbf{x}_{0}\right\|^{2} / 2 \sigma^{2}} \\
u_{2}(t=0, \mathbf{x})=0.001\left(x-x_{0}\right) \frac{1}{\sigma \sqrt{\pi}} e^{-\left\|\mathbf{x}-\mathbf{x}_{0}\right\|^{2} / 2 \sigma^{2}} \\
p(t=0, \mathbf{x})=1
\end{array}\right.
$$

on the domain $\Omega=[0,2]^{2}$, with $\sigma=0.4,\left(x_{0}, y_{0}\right)=(0.5,0.5)$ and the ideal gas law $(\gamma=1.4)$. As in the previous case the aim is to capture the slow transport dynamics of the density. Moreover we would like to evaluate the preservation of the divergence free property on the velocity field. As time evolves the solution behaves as follows: the velocity remains constant in time and the density is simply advected at velocity $\boldsymbol{u}(t, \boldsymbol{x})=\boldsymbol{u}(t=0, \boldsymbol{x})=\left(u_{1}(t=0, \boldsymbol{x}), u_{2}(t=0, \boldsymbol{x})\right)$. The 
Mach number is about $10^{-3}$. The final time is $T_{f}=1$ and we consider $200 \times 200$ cells. For the relaxation parameters we take

$$
\begin{aligned}
\text { Semi-implicit Suliciu: } & \lambda_{c}=1.8, \lambda_{a}=1.4 \\
\text { Semi-implicit two-speed: } & \lambda_{c}=3.2, \lambda_{a}=2.7, \phi=0.099 .
\end{aligned}
$$

In Table 17 we observe that the explicit (Rusanov) scheme is not accurate on the density as in the one dimensional test-cases. In the $2 \mathrm{~d}$ case this scheme is also not accurate on the velocity due to a numerical diffusion error proportional to $\Delta \boldsymbol{u}$. The explicit (FVS) scheme behaves similarly.

Then we note that the semi-implicit schemes are able to accurately capture both the density and the velocity since they use central fluxes in the implicit acoustic part of the scheme. The SI two-speed scheme with the FVS flux is the most accurate. These results are obtained with a 2000 times larger timestep than the one of the explicit scheme and 4 times larger than the one of the SI Suliciu scheme. This demonstrates the great benefit of the SI two-speed method for computing steady low Mach flows.

In Figure 4 are plotted the density and the 2D norm of the velocity field at final time in the 2D plots. These two quantities should remain close to invariant over time and there is no visible dissipation of the solution. In 1D plots are compared the initial data in red and the solution at time $t=1$. The results clearly show that the SI two-speed (wih FVS) has less numerical diffusion for both density and velocity.

\begin{tabular}{|c|c|c|c|c|c|}
\cline { 2 - 6 } \multicolumn{1}{c|}{} & \multicolumn{2}{c|}{ Explicit } & SI Suliciu & \multicolumn{2}{c|}{ SI two-speed } \\
\cline { 2 - 6 } \multicolumn{1}{c|}{} & Rusanov & FVS & Rusanov & Rusanov & FVS \\
\hline$\rho$ & 0.25 & $9.9 E^{-5}$ & $2.7 E^{-4}$ & $3.8 E^{-4}$ & $3.8 E^{-5}$ \\
\hline $\boldsymbol{u}$ & 0.62 & 0.33 & $\mathrm{x} 4.1 E^{-3}$ & $2.1 E^{-4}$ & $1.7 E^{-4}$ \\
\hline$p$ & $3.9 E^{-7}$ & $2.4 E^{-7}$ & $1.45 E^{-6}$ & $1.6 E^{-7}$ & $1.6 E^{-7}$ \\
\hline$\Delta t$ & $1.3 E^{-3}$ & $1.3 E^{-3}$ & $1.0(1.1 \mathrm{max})$ & $1.0(4.3 \mathrm{max})$ & $1.0(4.3 \mathrm{max})$ \\
\hline
\end{tabular}

Table 17: (Smooth contact wave, divergence free velocity, 2d) Numerical errors ( $L^{2}$ norm) and timestep $\Delta t$ for the different schemes.

\subsection{Gresho vortex (2d)}

We now consider the Gresho vortex test-case which is a classical test case for low Mach flows. Contrary to the previous test-case, the initial pressure involves a non-homogeneous spatial per- 

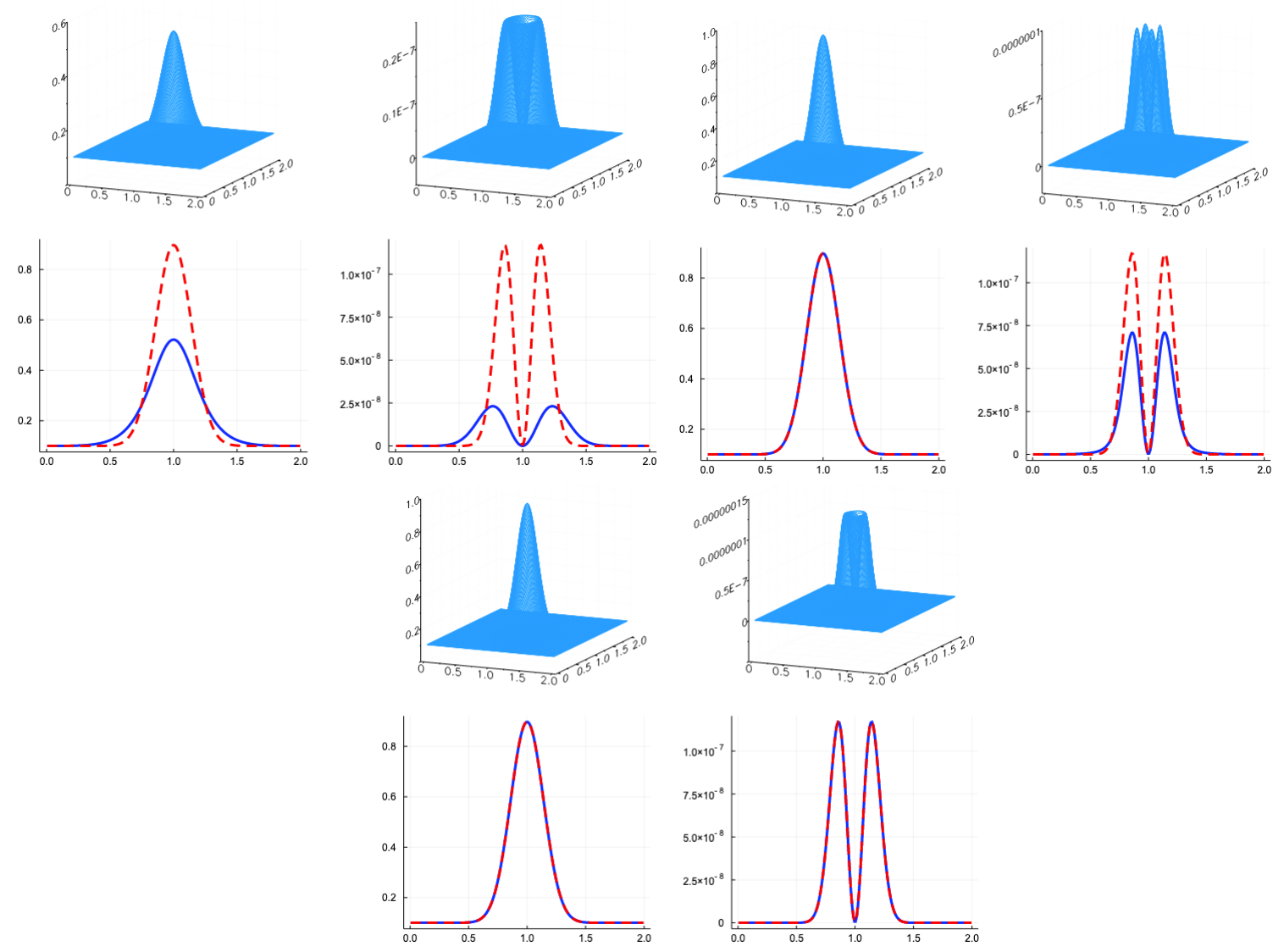

Figure 4: (Smooth contact wave, divergence free velocity, 2d) Density and 2D norm of the velocity for the different schemes (Explicit Rusanov scheme, Explicit Lagrange-Remap, Semi Implicit relaxation). $2 \mathrm{D}$ plots: at time $T_{f}=1$. 1D plots: one dimensional cut of the solution at initial data in red, at time $T_{f}=1$ in blue.

turbation. The initial condition is given by

$$
\begin{gathered}
\rho(t=0, \boldsymbol{x})=1, \quad \boldsymbol{u}(t=0, \boldsymbol{x})= \begin{cases}5 r \mathbf{e}_{\phi}, & \text { for } r \leqslant 0.2, \\
(2-5 r) \mathbf{e}_{\phi}, & \text { for } 0.2<r \leqslant 0.4, \\
\mathbf{0}, & \text { for } r>0.4,\end{cases} \\
p(t=0, \boldsymbol{x})= \begin{cases}p_{c}+\frac{25}{2} r^{2}, & \text { for } r \leqslant 0.2, \\
p_{c}+4 \ln (5 r)+4-20 r+\frac{25}{2} r^{2}, & \text { for } 0.2<r \leqslant 0.4, \\
p_{c}+4 \ln 2-2, & \text { for } r>0.4,\end{cases}
\end{gathered}
$$

on the domain $\Omega=[0,1]^{2}$ with $r=\left\|\mathbf{x}-\mathbf{x}_{0}\right\|, \mathbf{x}_{0}=(0.5,0 ; 5)$ and $p_{c}=\frac{1}{\gamma M_{0}^{2}}-\frac{1}{2}$ with a perfect gas law with $\gamma=1.4$. The parameter $M_{0}$ denotes the Mach number and will take the values 0.5 , 
$0.1,0.01$ or 0.001 . As in the test-case of weakly colliding pulses we can remove the constant $1 / \gamma M_{0}^{2}$ in the pressure law and recover a stiffened gas law with bounded initial pressure. Then since $\nabla \cdot \boldsymbol{u}^{0}=0$ the initial data is well-prepared. Indeed the above initial data is a steady solution to the incompressible Euler system, as well as a steady solution to the compressible system.

We compare the SI two-speed scheme with the classical Rusanov scheme with $120 \times 120$ cells. As usual we take $\theta=1$ and $\omega=1$, and for the relaxation we take $\phi=0.98$.

Figure 5 (resp. 6) shows $2 \mathrm{D}$ plots of the norm of the velocity at final time $T_{f}=1$ as well as $1 \mathrm{D}$ cuts of the final density and initial density obtained with the explicit Lagrange-Remap explicit scheme (resp. SI two-speed scheme with the FVS flux) for different Mach numbers. We observe that with a larger time-step independent of the Mach number, the SI two-speed scheme preserves the structure of the velocity field, contrary to the explicit scheme. More precisely, the accuracy of the classical scheme decreases with the Mach number and the CPU cost increases since the timestep decreases. Concerning the SI two-speed scheme, the accuracy on the density is almost the same whatever the Mach number is, and the CPU cost remains almost constant as the timestep is not modified. The CPU cost can be still impacted by the condition number of the implicit operator, but as already mentioned, a multigrid preconditioning technique could mitigate this weakness.

In Figure 7 are plotted the three variables (density, norm of the velocity and pressure) obtained with the SI two-speed scheme, for $M=0.01$ and for two different mesh sizes: $120 \times 120$ cells and $240 \times 240$ cells. We clearly observe the convergence on the three variables. To complete the analysis of this test case we propose to focus on the pressure. On Figure 7 we observe that the scheme captures the pressure perturbation but we also see oscillations on coarse grids. These oscillations come from the central derivative used in the implicit spatial part and the fact that the coefficient in front of this central derivative $\left(\lambda_{a} \Delta t\right)$ is very large. Indeed in this case the implicit operator is close to discrete divergence and gradient operators that admit spurious modes. If we decrease the Mach number the oscillations increase. Compared to the total size of the pressure the error remains small and these do not induce visible oscillations on the velocity. In order to avoid this problem of oscillations on the pressure, we shall investigate in the future structure-preserving methods for the implicit part, such as staggered finite differences.

\subsection{Kelvin-Helmholtz instability (2d)}

Finally, we consider a Kelvin-Helmholtz test case as proposed in [24]. The initial data is given by

$$
\rho(t=0, \boldsymbol{x})= \begin{cases}\rho_{1}-\rho_{m} \exp \left(\frac{y-0.25}{L}\right), & \text { if } 0 \leq y<0.25, \\ \rho_{2}+\rho_{m} \exp \left(\frac{0.25-y}{L}\right), & \text { if } 0.25 \leq y<0.5, \\ \rho_{2}+\rho_{m} \exp \left(\frac{y-0.75}{L}\right), & \text { if } 0.5 \leq y<0.75, \\ \rho_{1}-\rho_{m} \exp \left(\frac{0.75-y}{L}\right), & \text { if } 0.75 \leq y<1,\end{cases}
$$

with $\rho_{1}=1, \rho_{2}=2, \rho_{m}=\frac{1}{2}\left(\rho_{1}-\rho_{2}\right)$ and $L=0.025$. The velocity $u_{x}(t=0, \boldsymbol{x})$ has the same structure as the density with $u_{1}=0.5, u_{2}=-0.5$ and the pressure is constant: $p(t=0, \boldsymbol{x})=$ $2.5 / M_{0}^{2}$. We consider a perturbation $u_{y}(t=0, \boldsymbol{x})=0.01 \sin (2 \pi k x)$ with $k \in \mathbb{N}^{*}$. 

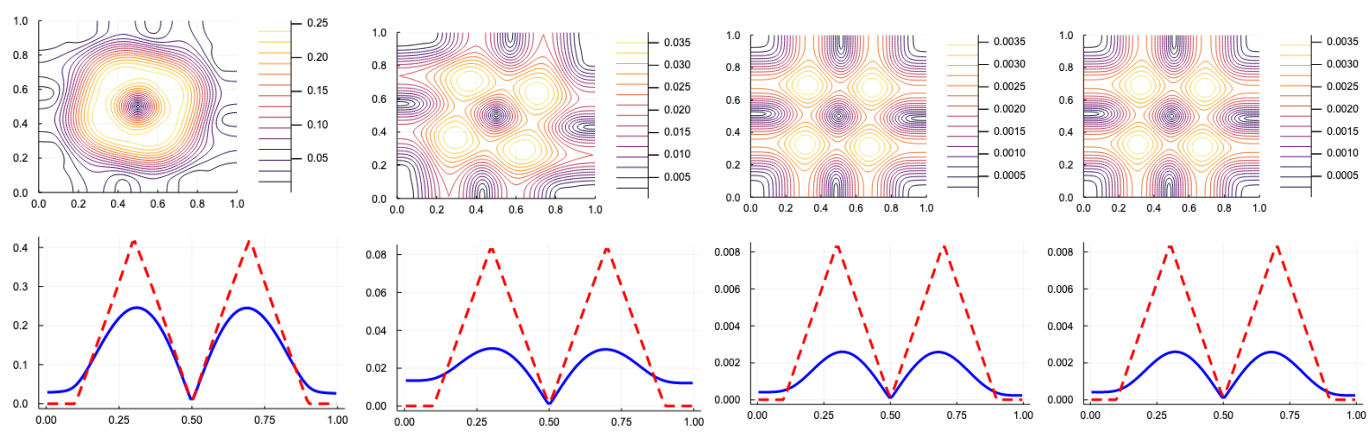

Figure 5: (Gresho vortex, explicit Lagrange+remap scheme) Norm of the velocity (2D plot) at final time $T f=1$ and initial (red) and final (blue) density for the explicit Rusanov scheme. From left to right: $M_{0}=0.5\left(\Delta t=1.4 E^{-3}\right), M_{0}=0.1\left(\Delta t=3.5 E^{-4}\right), M_{0}=0.01\left(\Delta t=3.5 E^{-5}\right)$, $M_{0}=0.001\left(\Delta t=3.5 E^{-6}\right)$.

First we compare the explicit Lagrange-Remap scheme with the results obtained with the SI two-speed relaxation scheme with FVS flux at time $T_{f}=3$ for $k=1$ (first mode) and $M_{0}=0.1$. On Figure 8 we remark that the explicit LR scheme with 360 cells by direction (second picture) and the SI two-speed scheme with 42 cells by direction (third picture) show similar results. With 120 cells, the SI two-speed scheme is able to capture the instability, which is completely smoothed out by the explicit scheme with the same resolution. This result shows the accuracy of the SI two-speed scheme compared to the explicit scheme. In addition, the timestep for the SI two-speed scheme is $3.5 E^{-3}$, it is larger than the one used for the explicit scheme, $1.0 E^{-4}$.

In Fig. 9 we show the results obtained with the SI two-speed scheme for $k=2$ and $M_{0}=0.01$ with grids of size $120 \times 120$ and $240 \times 240$. We observe that the scheme is still accurate when the Mach number is smaller. Moreover, for the $120 \times 120$ grid size, the timestep is identical to the one used for the Mach number $M_{0}=0.1$. This is made possible by a stability condition that is independent of the Mach number.

\section{Conclusion}

We have introduced a semi-implicit two-speed relaxation scheme to solve the compressible Euler equations in the low Mach regime. The scheme is based mainly on two ingredients. The first is to use a relaxation system with two speeds, already introduced in [14] in the barotropic case, that generalizes the Suliciu system. It is entropy satisfying and has a numerical viscosity welladapted to low Mach flows. Having two speeds enables us to optimize their choice. The second ingredient is a dynamical Mach number dependent splitting, similar to the one proposed in [6], but here at the level of the relaxation system. Stability conditions define the range of admissible relaxation and splitting parameters. We then resolve separately the advection part of the splitting by an explicit method, and the acoustic part by an implicit method. The relaxation speeds are chosen so that the implicit system fully linearizes the acoustics and requires just to invert an elliptic operator with constant coefficients. 

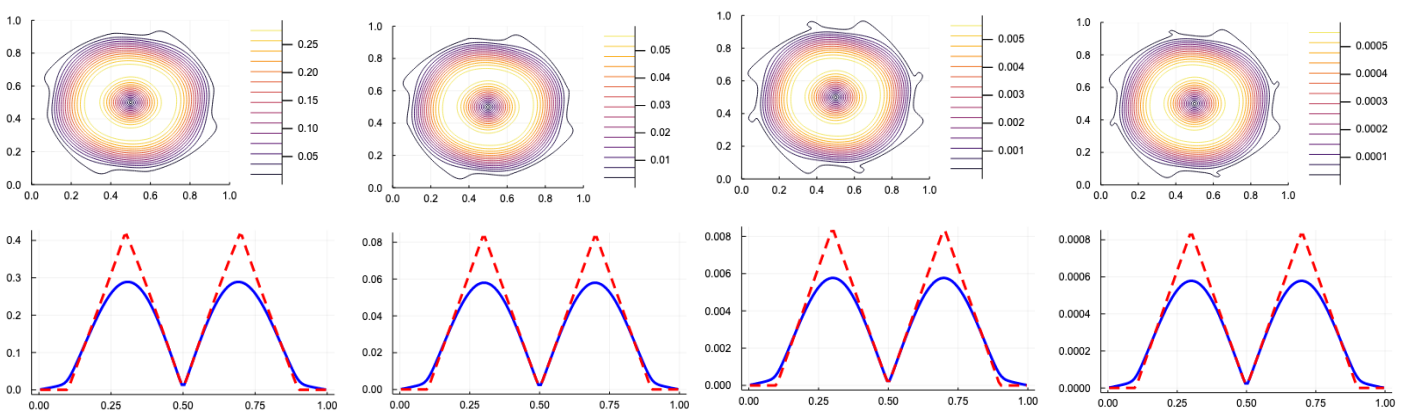

Figure 6: (Gresho vortex, SI two-speed. Norm of the velocity (2D plot) at final time $T_{f}=1$ and initial (red) and final (blue) density for the SI two-speed scheme. From left to right: $M=0.5$ $\left(\lambda_{c}=2.2, \lambda_{a}=1.8\right), \Delta t=2.5 E^{-3}, M=0.1\left(\lambda_{c}=11, \lambda_{a}=8.5\right), \Delta t=2.5 E^{-3}, M=0.01$ $\left(\lambda_{c}=120, \lambda_{a}=100\right), \Delta t=2.5 E^{-3}, M=0.001\left(\lambda_{c}=1150, \lambda_{a}=950\right), \Delta t=2.5 E^{-3}$.
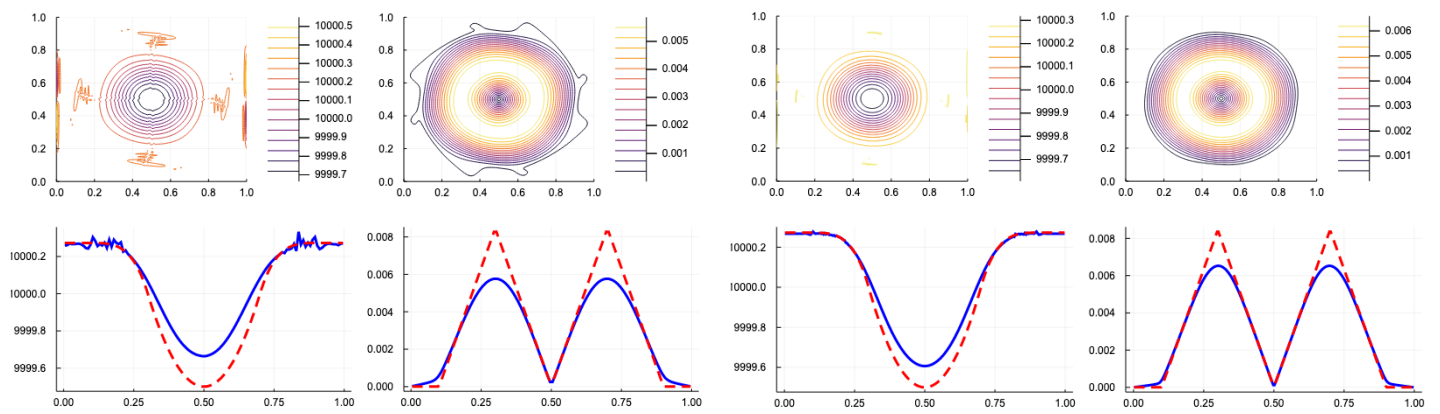

Figure 7: (Gresho vortex, SI two-speed) Density and norm of the velocity at time $T_{f}=1$ (2D plots and initial (red) / final (blue) density, velocity, pressure (1D cuts). Left: $120 \times 120$ cells. Right: $240 \times 240$ cells. $M_{0}=0.01$.

The numerical results show that the scheme is able to capture the slow scale of the low Mach dynamics, that is the leading incompressible Euler equations, with large timesteps adapted to the slow scale and not to the fast one. Moreover it is able to compute transient acoustic waves with a good accuracy relatively to the chosen timestep. The property to have a linear system with constant coefficients allows fast computations since the there is no matrix assembling at each timestep, and the condition number is not worsened by the space variations. Our method compares favorably with respect to other tested methods, in particular when strong relative variations of density occur. Although the implicit part of the scheme uses a timestep that is too large compared to the scale of the fast acoustic dynamics, it would be interesting to improve the accuracy of the fast acoustic dynamics. For doing that second-order schemes should be considered, but this extension is still difficult. Indeed the implicit second-order scheme in time and space generates oscillations that can trigger instabilities. Future work will be dedicated to this real challenge. One possible way could be to apply the strategy developed in [18]. Such a 

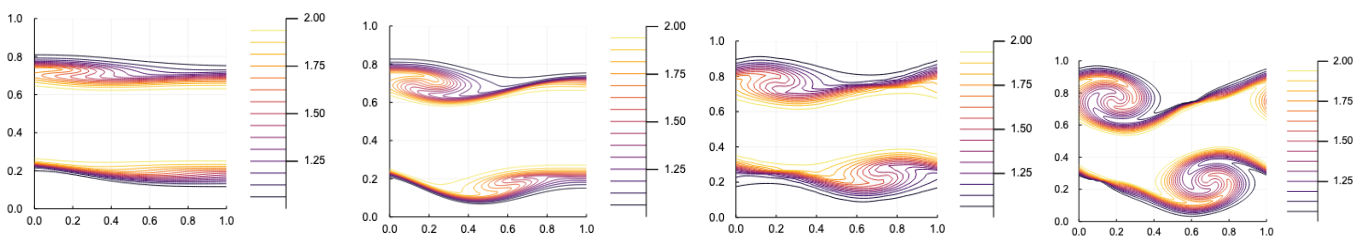

Figure 8: (Kelvin-Helmholtz) Density at time $T_{f}=3, k=1, M_{0}=0.1$. Explicit LagrangeRemap scheme with $120 \times 120$ (left) and $360 \times 360$ cells (middle left), SI two-speed relaxation scheme $\left(\lambda_{c}=18, \lambda_{a}=15, \phi=0.98\right)$ with $42 \times 42$ (middle right) and $120 \times 120$ cells (right).
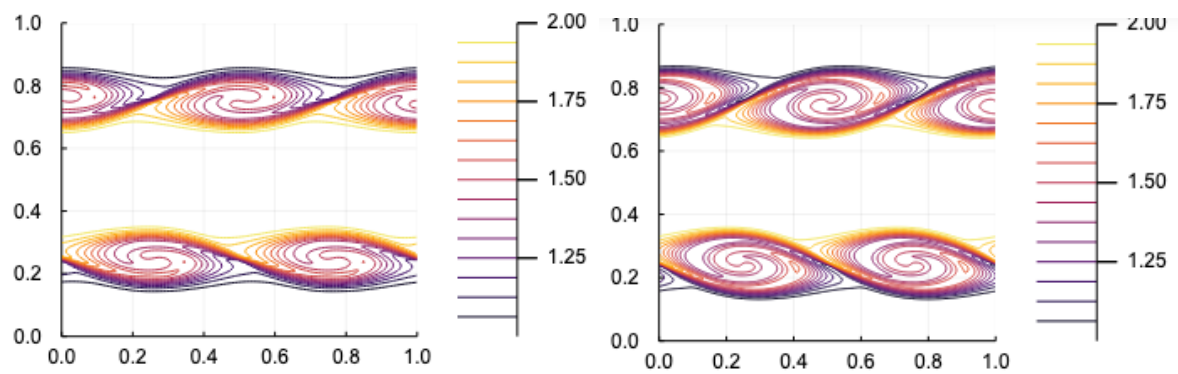

Figure 9: (Kelvin-Helmholtz) Density at time $T_{f}=3, k=2, M_{0}=0.01$ with SI two-speed relaxation scheme $\left(\lambda_{c}=180, \lambda_{a}=150, \phi=0.98\right)$. Left: $120 \times 120$ cells. Right: $240 \times 240$ cells.

method could also be very useful for solving hyperbolic problems for which there exists a slow scale dynamics around an equilibrium, with large timesteps. This kind of problem occurs for the Euler or Shallow water equations in the low-Mach regime with external source terms or in the Magneto-HydroDynamics (MHD) equations around a plasma equilibrium.

\section{Appendix}

\section{A Scheme based on the Suliciu relaxation system}

We give here the details of the method proposed in Subsection 2.2, that is a variant of the one of [6]. We split the convection step, the acoustic step and the relaxation step as (14), (15), (16). Each substep is performed as follows. 


\section{A.1 Convection step}

We use the finite volume scheme

$$
\left\{\begin{array}{l}
\rho_{j}^{n+1}-\rho_{j}^{n}+\frac{\Delta t}{\Delta x}\left(F_{j+\frac{1}{2}}^{\rho}-F_{j-\frac{1}{2}}^{\rho}\right)=0 \\
(\rho u)_{j}^{n+1}-(\rho u)_{j}^{n}+\frac{\Delta t}{\Delta x}\left(F_{j+\frac{1}{2}}^{\rho u}-F_{j-\frac{1}{2}}^{\rho u}\right)=0 \\
E_{j}^{n+1}-E_{j}^{n}+\frac{\Delta t}{\Delta x}\left(F_{j+\frac{1}{2}}^{E}-F_{j-\frac{1}{2}}^{E}\right)=0 \\
(\rho \Pi)_{j}^{n+1}-(\rho \Pi)_{j}^{n}+\frac{\Delta t}{\Delta x}\left(F_{j+\frac{1}{2}}^{\rho \Pi}-F_{j-\frac{1}{2}}^{\rho \Pi}\right)=0
\end{array}\right.
$$

with the local Lax- Friedrichs flux

$$
\left\{\begin{array}{l}
F_{j+\frac{1}{2}}^{\rho}=\frac{1}{2}\left((\rho u)_{j}+(\rho u)_{j+1}\right)-\frac{1}{2} S_{j+\frac{1}{2}}\left(\rho_{j+1}-\rho_{j}\right), \\
F_{j+\frac{1}{2}}^{\rho u^{2}}=\frac{1}{2}\left(\left(\rho u^{2}+\mathcal{E}^{2}(t) \Pi\right)_{j}+\left(\rho u^{2}+\mathcal{E}^{2}(t) \Pi\right)_{j+1}\right)-\frac{1}{2} S_{j+\frac{1}{2}}\left((\rho u)_{j+1}-(\rho u)_{j}\right), \\
F_{j+\frac{1}{2}}^{E}=\frac{1}{2}\left(\left(E u+\mathcal{E}^{2}(t) \Pi u\right)_{j}+\left(E u+\mathcal{E}^{2}(t) \Pi u\right)_{j+1}\right)-\frac{1}{2} S_{j+\frac{1}{2}}\left(E_{j+1}-E_{j}\right), \\
\left.F_{j+\frac{1}{2}}^{\rho \Pi^{2}}=\frac{1}{2}\left(\left(\rho \Pi u+\lambda_{c}^{2} u\right)_{j}+\left(\rho \Pi u+\lambda_{c}^{2} u\right)_{j+1}\right)-\frac{1}{2} S_{j+\frac{1}{2}}(\rho \Pi)_{j+1}-(\rho \Pi)_{j}\right),
\end{array}\right.
$$

with $S_{j+\frac{1}{2}} \geq \max \left(\left|u_{j}+\mathcal{E}(t) \frac{\lambda_{c}}{\rho_{j}}\right|,\left|u_{j}-\mathcal{E}(t) \frac{\lambda_{c}}{\rho_{j}}\right|,\left|u_{j+1}+\mathcal{E}(t) \frac{\lambda_{c}}{\rho_{j+1}}\right|,\left|u_{j+1}-\mathcal{E}(t) \frac{\lambda_{c}}{\rho_{j+1}}\right|\right)$. The associated CFL condition is then $S_{j+\frac{1}{2}} \Delta t \leq \Delta x$. We observe that for the original model the diffusion parameter $S$ is $\max (|u-c|,|u+c|)$, whereas here we replace $c$ by $\mathcal{E}(t) \frac{\lambda_{c}}{\rho}$ where $\lambda_{c}$ has to be large enough in terms of $\rho c$. We see that when the density admits a strong gradient and is close to zero somewhere in the domain, the new coefficient $\mathcal{E}(t) \frac{\lambda_{c}}{\rho}$ can be very large compared to the classical one. Consequently we get a more diffusive method in this case (strong density gradient and small values of the density), that is stable under a stronger CFL condition. This is however softened by the factor $\mathcal{E}(t)$.

\section{A.2 Acoustic step}

The acoustic step (15) is performed by the implicit time discretization (19). The result is that we compute the variables one after the other. We have $\rho^{n+1}=\rho^{n}$ and we compute $u^{n+1}$ via the elliptic equation

$$
u^{n+1}-\Delta t^{2}\left(1-\mathcal{E}^{2}(t)\right)^{2} \frac{1}{\rho^{n}} \partial_{x}\left(\frac{\lambda_{a}^{2}}{\rho^{n}} \partial_{x} u^{n+1}\right)=u^{n}-\Delta t\left(1-\mathcal{E}^{2}(t)\right) \frac{1}{\rho^{n}} \partial_{x} \Pi^{n} .
$$

Then we set

$$
\Pi^{n+1}=\Pi^{n}-\Delta t\left(1-\mathcal{E}^{2}(t)\right) \frac{\lambda_{a}^{2}}{\rho^{n}} \partial_{x} u^{n+1}=0
$$

and

$$
E^{n+1}=E^{n}-\Delta t\left(1-\mathcal{E}^{2}(t)\right) \partial_{x}\left(\Pi^{n+1} u^{n+1}\right) .
$$

In these equations we use centered finite differences in space. 


\section{A.3 Relaxation step}

The relaxation step is performed classically by

$$
\left\{\begin{array}{l}
\rho^{n+1}=\rho, \\
(\rho u)^{n+1}=\rho u, \\
E^{n+1}=E, \\
\Pi^{n+1}=\Pi+\omega(p-\Pi),
\end{array}\right.
$$

with $1 \leq \omega \leq 2$ to allow over-relaxation [17].

\section{B Numerical fluxes for the convection step of the two speed relax- ation scheme}

We give here the different numerical fluxes used for the convection step of our scheme, as proposed in Subsection 4.3.1.

\section{B.1 Local Lax Friedrichs flux}

The first possible choice is simple and classical: the local Lax-Friedrichs numerical flux, which is the sum of a centered term and a diagonal viscosity term with the local maximum eigenvalue as coefficient. The numerical flux for (68) is thus given by

$$
\left\{\begin{aligned}
F_{j+\frac{1}{2}}^{\rho} & =\frac{1}{2}\left((\rho v)_{j}+(\rho v)_{j+1}\right)-\frac{1}{2} S_{j+\frac{1}{2}}\left(\rho_{j+1}-\rho_{j}\right), \\
F_{j+\frac{1}{2}}^{\rho u} & =\frac{1}{2}\left(\left(\rho u v+\mathcal{E}^{2}(t) \Pi\right)_{j}+\left(\rho u v+\mathcal{E}^{2}(t) \Pi\right)_{j+1}\right)-\frac{1}{2} S_{j+\frac{1}{2}}\left((\rho u)_{j+1}-(\rho u)_{j}\right), \\
F_{j+\frac{1}{2}}^{E} & =\frac{1}{2}\left(\left(E v+\mathcal{E}^{2}(t) \Pi v\right)_{j}+\left(E v+\mathcal{E}^{2}(t) \Pi v\right)_{j+1}\right)-\frac{1}{2} S_{j+\frac{1}{2}}\left(E_{j+1}-E_{j}\right) \\
F^{\pi} & =\frac{\phi \lambda_{c}^{2}}{2}\left(v_{j}+v_{j+1}\right)-\frac{1}{2} S_{j+\frac{1}{2}}\left(\Pi_{j+1}-\Pi_{j}\right), \\
F_{j+\frac{1}{2}}^{v} & =\frac{1}{2 \phi} \mathcal{E}^{2}(t)\left(\Pi_{j}+\Pi_{j+1}\right)-\frac{1}{2} S_{j+\frac{1}{2}}\left(v_{j+1}-v_{j}\right)
\end{aligned}\right.
$$

with

$$
S_{j+\frac{1}{2}}=\max \left(\left|v_{j}-\mathcal{E}(t) \lambda_{c}\right|,\left|v_{j}+\mathcal{E}(t) \lambda_{c}\right|,\left|v_{j+1}-\mathcal{E}(t) \lambda_{c}\right|,\left|v_{j+1}+\mathcal{E}(t) \lambda_{c}\right|\right),
$$

and for the nonconservative fluxes we use

$$
\begin{aligned}
& F_{j}^{\pi}=\max \left(v_{j}, 0\right)\left(\Pi_{j}-\Pi_{j-1}\right)+\min \left(v_{j}, 0\right)\left(\Pi_{j+1}-\Pi_{j}\right), \\
& F_{j}^{v}=\max \left(v_{j}, 0\right)\left(v_{j}-v_{j-1}\right)+\min \left(v_{j}, 0\right)\left(v_{j+1}-v_{j}\right) .
\end{aligned}
$$

The associated CFL condition is then $S_{j+\frac{1}{2}} \Delta t \leq \Delta x$.

\section{B.2 Linear acoustic flux splitting (FVS)}

The idea of [25], [9] introduced previously in [23] consists in splitting the fluxes in several terms related to acoustic waves. Adapting the idea we propose to split (47) in three parts: conservative 
terms, advection of pressure and velocity terms and acoustic terms:

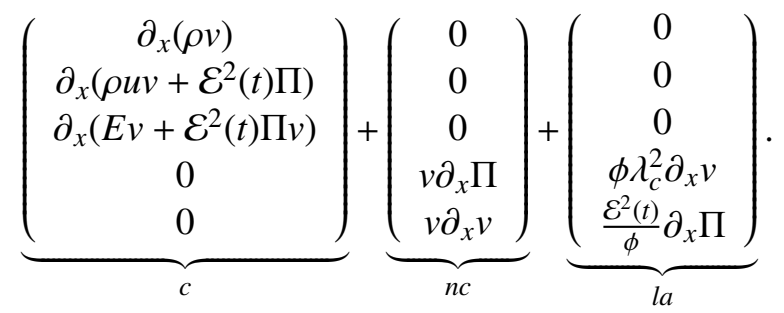

These are called conservative terms "c", nonconservative terms "nc" and linear acoustic terms "la'. Let us describe the schemes for each part of this flux splitting. For the nonconservative part we use the nonconservative numerical fluxes (87). For the linear acoustic part we propose to use classical upwind acoustic fluxes. For that we diagonalize the linear acoustic part to obtain

$$
\left\{\begin{array}{c}
\partial_{t}\left(\frac{\phi \lambda_{c} v}{\mathcal{E}(t)}-\Pi\right)-\mathcal{E}(t) \lambda_{c} \partial_{x}\left(\frac{\phi \lambda_{c} v}{\mathcal{E}(t)}-\Pi\right)=0 \\
\partial_{t}\left(\frac{\phi \lambda_{c} v}{\mathcal{E}(t)}+\Pi\right)+\mathcal{E}(t) \lambda_{c} \partial_{x}\left(\frac{\phi \lambda_{c} v}{\mathcal{E}(t)}+\Pi\right)=0
\end{array}\right.
$$

We apply the upwind scheme to each of these equations and get

$$
\left\{\begin{array}{l}
\left(\frac{\phi \lambda_{c} v}{\mathcal{E}(t)}-\Pi\right)_{j+\frac{1}{2}}=\left(\frac{\phi \lambda_{c} v}{\mathcal{E}(t)}-\Pi\right)_{j+1}, \\
\left(\frac{\phi \lambda_{c} v}{\mathcal{E}(t)}+\Pi\right)_{j+\frac{1}{2}}=\left(\frac{\phi \lambda_{c} v}{\mathcal{E}(t)}+\Pi\right)_{j} .
\end{array}\right.
$$

We thus obtain conservative fluxes for which $v$ and $\pi$ are evaluated according to

$$
\left\{\begin{array}{l}
v_{j+\frac{1}{2}}=\frac{1}{2}\left(v_{j}+v_{j+1}\right)-\frac{\mathcal{E}(t)}{2 \phi \lambda_{c}}\left(\Pi_{j+1}-\Pi_{j}\right), \\
\Pi_{j+\frac{1}{2}}=\frac{1}{2}\left(\Pi_{j}+\Pi_{j+1}\right)-\frac{\phi \lambda_{c}}{2 \mathcal{E}(t)}\left(v_{j+1}-v_{j}\right) .
\end{array}\right.
$$

To finish we have to choose a scheme for the conservative part. We propose to use the values (88) for the relaxed pressure terms. For the advection at velocity $v$ we use an upwind scheme with the velocity given by (88). Finally, gathering the fluxes for the three parts we obtain the global fluxes

$$
\left\{\begin{aligned}
F_{j+\frac{1}{2}}^{\rho} & =\frac{v_{j+\frac{1}{2}}}{2}\left(\rho_{j}+\rho_{j+1}\right)-\frac{\left|v_{j+\frac{1}{2}}\right|}{2}\left(\rho_{j+1}-\rho_{j}\right), \\
F^{\rho u} & =\frac{v_{j+\frac{1}{2}}}{2}\left((\rho u)_{j}+(\rho u)_{j+1}\right)-\frac{\left|v_{j+\frac{1}{2}}\right|}{2}\left((\rho u)_{j+1}-(\rho u)_{j}\right)+\mathcal{E}^{2}(t) \Pi_{j+\frac{1}{2}}, \\
F_{j+\frac{1}{2}}^{E} & =\frac{v_{j+\frac{1}{2}}}{2}\left(E_{j}+E_{j+1}\right)-\frac{\left|v_{j+\frac{1}{2}}\right|}{2}\left(E_{j+1}-E_{j}\right)+\mathcal{E}^{2}(t) \Pi_{j+\frac{1}{2}} v_{j+\frac{1}{2}}, \\
F^{\pi} & =\phi \lambda_{c}^{2} v_{j+\frac{1}{2}}, \\
F_{j+\frac{1}{2}}^{v} & =\frac{\mathcal{E}^{2}(t)}{\phi} \Pi_{j+\frac{1}{2}},
\end{aligned}\right.
$$

where $v_{j+\frac{1}{2}}, \Pi_{j+\frac{1}{2}}$ are defined by (88). These have to be put in (68) with the nonconservative fluxes (87). At the end the final flux is similar to the flux used in [10] where we advect the conservative quantities with the flux obtained for acoustics. 


\section{B.3 Godunov scheme}

The Godunov scheme can used to solve system $(C)$ i.e. (47). Indeed the system has all its characteristic fields linearly degenerate, thus we can compute the solution to the Riemann problem explicitly. As mentioned previously, (47) identifies with the original relaxation system (20) without right-hand side, in which we replace $\Pi$ by $\widetilde{\Pi}=\mathcal{E}^{2}(t) \Pi, a$ by $\widetilde{a}=\mathcal{E}(t) \sqrt{\chi_{c}} a$ and $b$ by $\widetilde{b}=\mathcal{E}(t) \sqrt{\chi_{c}} b$.

Let us therefore consider (20) (without right-hand side relaxation terms). This system identifies with the system of [14] except for the addition of a new unknown $E$, or equivalently $e$ or $\widehat{e}$, that satisfies (41), (42). We deduce the solution to the Riemann problem as follows. Consider left and right values of $\rho, u, e, \Pi, v, a, b$. Then the solution has three speeds

$$
\sigma^{L}=v^{L}-\frac{a^{L}}{\rho^{L}}, \quad \sigma^{\sharp}=v^{\sharp}, \quad \sigma^{R}=v^{R}+\frac{a^{R}}{\rho^{R}},
$$

where $v^{\sharp}$ is defined below. They are correctly ordered $\left(\sigma^{L}<\sigma^{\sharp}<\sigma^{R}\right)$ as soon as the subcharacteristic condition is satisfied. These speeds determine two regions $\sigma^{L}<x / t<\sigma^{\sharp}$ and $\sigma^{\sharp}<x / t<\sigma^{R}$ in which the solution takes values denoted by $L \sharp$ and $R \sharp$ respectively. These intermediate states are computed by

$$
\begin{aligned}
& \Pi^{L \sharp}=\Pi^{R \sharp} \equiv \Pi^{\sharp}=\frac{b^{R} \Pi^{L}+b^{L} \Pi^{R}+b^{L} b^{R}\left(v^{L}-v^{R}\right)}{b^{L}+b^{R}}, \\
& v^{L \sharp}=v^{R \sharp} \equiv v^{\sharp}=\frac{b^{L} v^{L}+b^{R} v^{R}+\Pi^{L}-\Pi^{R}}{b^{L}+b^{R}}, \\
& \frac{1}{\rho^{L \sharp}}=\frac{1}{\rho^{L}}+\frac{b^{R}\left(v^{R}-v^{L}\right)+\Pi^{L}-\Pi^{R}}{a^{L}\left(b^{L}+b^{R}\right)}, \\
& \frac{1}{\rho^{R \sharp}}=\frac{1}{\rho^{R}}+\frac{b^{L}\left(v^{R}-v^{L}\right)+\Pi^{R}-\Pi^{L}}{a^{R}\left(b^{L}+b^{R}\right)}, \\
& u^{L \sharp}=u^{L}+\frac{b^{L}}{a^{L}\left(b^{L}+b^{R}\right)}\left(b^{R}\left(v^{R}-v^{L}\right)+\Pi^{L}-\Pi^{R}\right), \\
& u^{R \sharp}=u^{R}+\frac{b^{R}}{a^{R}\left(b^{L}+b^{R}\right)}\left(b^{L}\left(v^{L}-v^{R}\right)+\Pi^{L}-\Pi^{R}\right), \\
& e^{L \sharp}=e^{L}+\frac{\left(\Pi^{\sharp}\right)^{2}-\left(\Pi^{L}\right)^{2}}{2 a^{L} b^{L}}+\frac{\left(v^{\sharp}-u^{L \sharp}\right)^{2}-\left(v^{L}-u^{L}\right)^{2}}{2\left(\frac{a^{L}}{b^{L}}-1\right)}, \\
& e^{R \sharp}=e^{R}+\frac{\left(\Pi^{\sharp}\right)^{2}-\left(\Pi^{R}\right)^{2}}{2 a^{R} b^{R}}+\frac{\left(v^{\sharp}-u^{R \sharp}\right)^{2}-\left(v^{R}-u^{R}\right)^{2}}{2\left(\frac{a^{R}}{b^{R}}-1\right)}, \\
& a^{L \sharp}=a^{L}, a^{R \star}=a^{R}, b^{L \star}=b^{L}, b^{R \star}=b^{R} .
\end{aligned}
$$

Since (20) is in conservative form for $\rho, \rho u, E$, the corresponding numerical fluxes are $\rho v$, $\rho u v+\Pi, E v+\Pi v$ evaluated on the $L, L \sharp, R \sharp, R$ states in accordance with the value $x / t=0$ (in other words we take the value $L$ if $0 \leq \sigma^{L}, L \sharp$ if $\sigma^{L} \leq 0 \leq \sigma^{\sharp}, R \sharp$ if $\sigma^{\sharp} \leq 0 \leq \sigma^{R}$, and $R$ if $\sigma^{R} \leq 0$ ). For the $\Pi$ and $v$ variables, a possible choice is to consider the variables 
$\rho \Pi / a b$ and $\rho v b / a$, that satisfy conservative equations. Their fluxes $\rho \Pi v / a b+v, \rho v^{2} b / a+\Pi$ are thus evaluated at $x / t=0$ as the other conservative variables. This allows to compute the updated average values of $\rho \Pi / a b$ and $\rho v b / a$. We can compute similarly the updated average values of $\rho / a b$ and $\rho b / a$, and define the new values of $\Pi$ and $v$ by taking the ratios of averages $(\rho \Pi / a b) /(\rho / a b)$ and $(\rho v b / a) /(\rho b / a)$. Notice that according to the description of Subsection 4.2, $a$ and $b$ are reinitialized after the convection step. In the previous description of the Riemann problem and numerical fluxes, $a, b$ are not yet reinitialized.

Another way of updating $\Pi$ and $v$ is to directly average in space the $\Pi$ and $v$ equations of (22). We then have nonconservative terms and we have to proceed carefully, as in [15, section 5.3].

For our problem (47) we replace in the previous formulas $\Pi$ by $\widetilde{\Pi}=\mathcal{E}^{2}(t) \Pi$, $a$ by $\widetilde{a}=$ $\mathcal{E}(t) \sqrt{\chi_{c}} a$ and $b$ by $\widetilde{b}=\mathcal{E}(t) \sqrt{\chi_{c}} b$. Then according to Subsection 4.2 , the values of $\widetilde{a}^{L}, \widetilde{a}^{R}, \widetilde{b}^{L}$, $\widetilde{b}^{R}$ are

$$
\widetilde{a}^{L}=\mathcal{E}(t) \rho^{L} \lambda_{c}, \quad \widetilde{a}^{R}=\mathcal{E}(t) \rho^{R} \lambda_{c}, \quad \widetilde{b}^{L}=\widetilde{b}^{R}=\mathcal{E}(t) \phi \lambda_{c} .
$$

The extreme propagation speeds (89) become

$$
\widetilde{\sigma}^{L / R}=v^{L / R} \mp \mathcal{E}(t) \lambda_{c} .
$$

\section{References}

[1] Christophe Berthon. Stability of the muscl schemes for the euler equations. Comm. Math. Sci, pages 133-158, 2005.

[2] C. Chalons, F. Coquel, and C. Marmignon. Well-balanced time implicit formulation of relaxation schemes for the Euler equations. SIAM J. Scient. Comput., 30(1):394-415, 2008.

[3] C. Chalons, F. Coquel, and C. Marmignon. Time-implicit approximation of the multipressure gas dynamics equations in several space dimensions. SIAM J. Numer. Anal., 48(5):1678-1706, 2010.

[4] D. A. Knoll, V. A. Mousseau, L. Chacon, and J. Reisner. Jacobian-free Newton-Krylov methods for the accurate time integration of stiff wave systems. J. Scient. Comput., 25(1):213-230, 2005.

[5] D. Coulette, E. Franck, P. Helluy, A. Ratnani, and E. Sonnendruecker. Implicit time schemes for compressible fluid models based on relaxation methods. Computers and fluids, 188:70-85, 2019.

[6] D. Iampietro, F. Daude, P. Galon, and J.-M. Hérard. A Mach-sensitive implicit-explicit scheme adapted to compressible multi-scale flows. J. Comput. Appl. Math., 340:122-150, 2018.

[7] P. J. Dellar. Incompressible limits of lattice Boltzmann equations using multiple relaxation times. J. Comput. Phys., 190(2):351 - 370, 2003. 
[8] P. J. Dellar. An interpretation and derivation of the lattice Boltzmann method using Strang splitting. Computers Math. Applic., 65(2):129 - 141, 2013. Special Issue on Mesoscopic Methods in Engineering and Science (ICMMES-2010, Edmonton, Canada).

[9] E. F. Toro and M. E. Vázquez-Cendón. Flux splitting schemes for the Euler equations. Computers and Fluids, 70:1-12, 2012.

[10] E. Franck and L. Mendoza. Finite volume scheme with local high order discretization of the hydrostatic equilibrium for the euler equations with external forces. Journal of Scientific Computing, 69:314-354, 2016.

[11] F. Berthelin and F. Bouchut. Kinetic invariant domains and relaxation limit from a BGK model to isentropic gas dynamics. Asymptotic Analysis, 31(2):153-176, 2002.

[12] F. Bouchut. Nonlinear stability of finite volume methods for hyperbolic conservation laws, and well-balanced schemes for sources. Frontiers in Mathematics. Birkhauser, 2004.

[13] F. Bouchut. A reduced stability condition for nonlinear relaxation to conservation laws. $J$. Hyp. Diff. Eq., 1(1):149-170, 2004.

[14] F. Bouchut, C. Chalons, and S. Guisset. An entropy satisfying two-speed relaxation system for the barotropic Euler equations. Application to the numerical approximation of low Mach number flows. Numer. Math., 2020.

[15] F. Bouchut, C. Klingenberg, and K. Waagan. A multiwave approximate Riemann solver for ideal MHD based on relaxation II - Numerical implementation with 3 and 5 waves. Numer. Math., 115(4):647-679, 2010.

[16] F. Bouchut, Y. Jobic, R. Natalini, R. Occelli, and V. Pavan. Second-order entropy satisfying BGK-FVS schemes for incompressible Navier-Stokes equations. SMAI J. comput. math., 4:1-56, 2018.

[17] F. Drui, E. Franck, P. Helluy, and L. Navoret. An analysis of over-relaxation in a kinetic approximation of systems of conservation laws. Comptes Rendus Mécanique, 347(3):259 $-269,2019$.

[18] G. Dimarco, R. Loubère, V. Michel-Dansac, and M-H. Vignal. Second order implicitexplicit total variation diminishing schemes for the Euler system in the low Mach regime. J. Comput. Phys., 372:178-201, 2018.

[19] Sigal. Gottlieb, Chi-Wang. Shu, and Eitan. Tadmor. Strong stability-preserving high-order time discretization methods. SIAM Review, 43(1):89-112, 2001.

[20] R. Klein. Semi-implicit extension of a Godunov-type scheme based on low Mach number asymptotics I: One-dimensional flow. J. Comput. Phys., 121(2):213 - 237, 1995.

[21] L. Boittin, F. Bouchut, M.-O. Bristeau, A. Mangeney, J. Sainte-Marie, and F. Souille. The Navier-Stokes system with temperature and salinity for free surface flows Part I: LowMach approximation \& layer-averaged formulation. 2018. 
[22] L. Chacon. An optimal parallel fully implicit Newton - Krylov solver for three-dimensional viscoresistive magnetohydrodynamics. Phys. Plasmas, 15:056103, 2008.

[23] M. S. Liou and C. J. Steffen. A new flux splitting scheme. J. Comput. Phys., 107:23-29, 1993.

[24] Miczek, F., Röpke, F. K., and Edelmann, P. V. F. New numerical solver for flows at various mach numbers. AEAA, 576:A50, 2015.

[25] P. Tiam Kapen and T. Ghislain. A new flux splitting scheme based on Toro-Vazquez and HLL schemes for the Euler equations. J. Comput. Meth. Phys., 2014:827034, 2014.

[26] S. Boscarino, G. Russo, and L. Scandurra. All Mach number second order semi-implicit scheme for the Euler equations of gas dynamics. J. Scient. Comput., 77(2):850-884, Nov 2018.

[27] S. Boscarino, Jing-Mei Qiu, G. Russo, and Tao Xiong. A high order semi-implicit IMEX WENO scheme for the all-Mach isentropic Euler system. J. Comput. Phys., 392:594-618, 2019.

[28] S. Jin and Z. Xin. The relaxation schemes for systems of conservation laws in arbitrary space dimensions. Commu. Pure appl. math., 48(3):235-276, 1995. 